\title{
Theoretical Investigations of Hydrogen Bonding Interactions of (E)-1-(1H-Benzo[d]imidazol-2-yl)-3- Phenylprop-2-en-1-one Momoners and Dimers: NBO, QTAIM and NCI Study
}

\author{
Adenidji Ganiyou ${ }^{1}$, Kicho Denis Yapo ${ }^{1,}$, , Doumadé Zon ${ }^{1,3}$, Mamadou Guy-Richard Kone ${ }^{2}$ \\ ${ }^{1}$ Training and Research Unit of Sciences of Structures, Matter and Technology (UFR SSMT), Laboratory of Organic Chemistry and Natural \\ Substances, Félix Houphouët-Boigny University, Abidjan, Côte d'Ivoire \\ ${ }^{2}$ Faculty of Fundamental and Applied Sciences (UFR SFA), Laboratory of Thermodynamics and Physico-Chemistry of the Middle, Nangui \\ Abrogoua University, Abidjan, Côte d'Ivoire \\ ${ }^{3}$ Department of Biochemistry-Genetics, Training and Research Unit in Biological Science, Peleforo Gon Coulibaly University, Korhogo, \\ Côte-d'Ivoire
}

Email address:

yapokicho@gmail.com (K. D. Yapo)

*Corresponding author

\section{To cite this article:}

Adenidji Ganiyou, Kicho Denis Yapo, Doumadé Zon, Mamadou Guy-Richard Kone. Theoretical Investigations of Hydrogen Bonding Interactions of (E)-1-(1H-Benzo[d]imidazol-2-yl)-3-Phenylprop-2-en-1-one Momoners and Dimers: NBO, QTAIM and NCI Study. Modern Chemistry. Vol. 7, No. 4, 2019, pp. 80-94. doi: 10.11648/j.mc.20190704.11

Received: August 19, 2019; Accepted: September 16, 2019; Published: October 9, 2019

\begin{abstract}
Hydrogen bonding is an essential interaction in nature and plays a crucial role in many formations of materials and biological processes, requiring a deeper understanding of its formation. Benzimidazole is an important structural unit found in a large number of natural and pharmacologically active molecules. In the present work, the electronic structures and properties and relatives stabilities of a series of (E)-1-(1H-benzo[d]imidazol-2-yl)-3-phenylprop-2-en-1-one monomers and dimers have been studied by density functional theory using B3LYP $6-31+G(d, p)$ calculation level. the strengths of the noncovalent interactions have been analyzed in terms of the QTAIM analysis, NCI analysis and natural bond orbital approaches. It was found that the dimers are formed by double $\mathrm{N}-\mathrm{H} \cdots \mathrm{O}$ hydrogen bond. QTAIM analysis proved the presence of intramolecular hydrogen bond in monomers and coexistence of intramolecular and intermolecular hydrogen bond in dimers. Frequency analysis show that intermolecular $\mathrm{N}-\mathrm{H} \cdots \mathrm{O}$ interactions are proper hydrogen bond while intramolecular $\mathrm{C}-\mathrm{H} \cdots \mathrm{N}, \mathrm{C}-$ $\mathrm{H} \cdots \mathrm{O}, \mathrm{C}-\mathrm{H} \cdots \mathrm{H}-\mathrm{C}$ interactions are improper hydrogen bond. $\mathrm{NBO}$ and $\mathrm{NCI}$ analyses confirm the existence of hydrogen bonds in the studied monomers and dimers. The presence of weakly electron acceptor group on benzene ring favor the total interaction energy of dimerization.
\end{abstract}

Keywords: NBO, QTAIM, NCI, Benzimidazole, Electron Density, Hydrogen Bond

\section{Introduction}

Benzimidazole (BZim) is a heterocyclic aromatic organic compound. It is an important drug and a preferred structure in medicinal chemistry [1-3]. The benzimidazole ring is one of the bioactive heterocyclic compounds that have a range of biological activities such as antivirals (anti-HIV), anticancer drugs, antibacterials, antifungals and many others [4-9]. Benzimidazole derivatives are associated with various types of pharmacokinetics and pharmacodynamics properties. The most important benzimidazole compound in nature is $\mathrm{N}$ ribosyldimethylbenzimidazole, which serves as an axial ligand of cobalt in vitamin B12 [10]. In addition, some benzimidazoles are used in coordination chemistry [11-14], optoelectronics [15].

The hydrogen bond is one of the fundamental interactions that plays a key role in many fields of chemistry, physics and biology [16-19]. It affects the stability of many important molecular structures such as water $[20,21]$ and DNA [22]. 
Knowledge of hydrogen bonding strength is essential for physical, chemical and biological applications [23]. In addition, a slight difference in molecular structure may play a role in the strength of hydrogen bonds $[24,25]$. In most cases, the formation of $X-H \cdots Y$ bonds leads to a weakening of the $\mathrm{X}-\mathrm{H}$ bond, accompanied by an elongation of the bond and a concomitant decrease of the vibrational stretching frequency of the $\mathrm{X}-\mathrm{H}$ bond relative to the noninteractive bond, these bonds are called proper or Red shifted hydrogen bond. However, there are also many examples in which the stretching frequency of the X-H bond increases with the contraction of the bond length during the formation of the hydrogen bond, these are improper or Blue shifted hydrogen bond [26].

The electronic foundations of the improper hydrogen bond were analyzed by Alabugin et al [27]. The structural reorganization of the $\mathrm{X}-\mathrm{H}$ bond observed for proper and improper hydrogen bonds results from the balance between hyperconjugation $\left(n_{X} \rightarrow \sigma_{X-H}^{*}\right)$ which weakens the $\mathrm{X}-\mathrm{H}$ bond and rehybridization which strengthens X-H bond [27]. Improper hydrogen bonds are observed when the hyperconjugative interaction is relatively low. When the hyperconjugative interaction is low and the hybrid orbital of the $\mathrm{X}$ atom of the $\mathrm{X}-\mathrm{H}$ bond is able to undergo hybridization, rehybridization prevails, resulting in a shortening of the $\mathrm{X}-\mathrm{H}$ bond and a blue shift of the $\mathrm{X}-\mathrm{H}$ vibrational stretching frequency. The presence of electron-rich centers $(\mathrm{N}, \mathrm{O})$ and hydrogen atoms linked by a covalent bond to nitrogen and carbon atoms offers the possibility of different types of noncovalent bonds such as $C-H \cdots N, C-H \cdots O, N-H \cdots O$ (Figure 1).

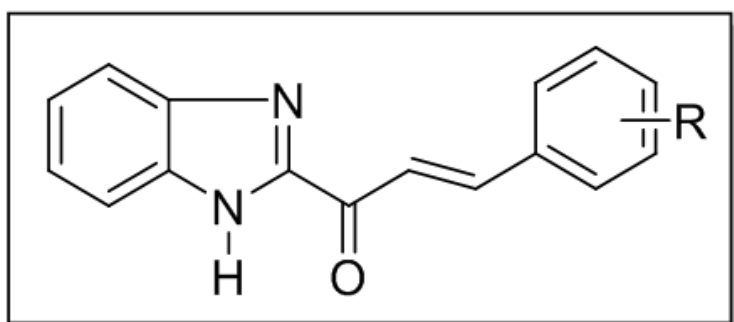

\begin{tabular}{lll}
\hline MONOMER & R & DIMER \\
\hline M1 & $\mathrm{H}$ & $D_{1}=M_{1} \cdots M_{1}$ \\
M2 & $4-\mathrm{C} 1$ & $D_{2}=M_{2} \cdots M_{2}$ \\
M3 & $4-\mathrm{F}$ & $D_{3}=M_{3} \cdots M_{3}$ \\
M4 & $4-\mathrm{NO}_{2}$ & $D_{4}=M_{4} \cdots M_{4}$ \\
M5 & $2-\mathrm{C} 1,5-\mathrm{NO}_{2}$ & $D_{5}=M_{5} \cdots M_{5}$ \\
\hline
\end{tabular}

Figure 1. Structure of M1-M5 monomers and D1-D5 dimers studied.

In this study, we applied quantum chemistry methods to determine the interaction energies and understand the nature of the different hydrogen bonds of BZims monomers and dimers. The topological properties, hyperconjugative interactions between donor and proton acceptor of different $X-H \cdots Y$ hydrogen bonds formed have been determined. The QTAIM analysis was used to assess the strength and nature of intramolecular and intermolecular hydrogen bonds. We also performed the NBO (natural bond orbital) and NCI (non-covalent interaction) analyses that suggest the existence of hydrogen bonds in the studied systems. Our calculations will give a theoretical overview on the possibility of dimerization of BZims.

\section{Materials, Methods and Parameters}

\subsection{Computational Methods and Materials}

The monomers M1-M5 and dimers D1-D5 (Figure 1) were optimized using B3LYP [28, 29] calculation level with a 6$31+\mathrm{G}(\mathrm{d}, \mathrm{p})$ basis set. The optimization was performed with Gaussian 09 [30] software on Intel i7 2600 processors. In a further step, the topological analysis of the electron density was performed from the "formatted checkpoint file" obtained by the DFT calculation with AIMALL software [31]. The NBO orbital analysis method [32] (NBO: Natural orbital bond) incorporated in the Gaussian 09 software was used for NBO analyses. NCI analysis was performed with Multiwfn software [33] and NCI analysis surfaces were represented with Chemcraft software [34].

\subsection{Interaction Energy}

The interaction energies of the complexes were determined by the difference between the total energy of the complex and the energies of the isolated monomers, then corrected by taking account the basic set superposition error (BSSE) using the Boys-Bernardi counterpoise procedure [35] and the ZPE:

$$
E_{\text {int }}=E^{\text {Dimer }}-\left(2 * E_{\text {monomer }}\right)+b s s e+\Delta Z P E
$$

With $\triangle Z P E=Z P E^{\text {Dimer }}-2 * Z P E^{\text {monomer }}$

\subsection{NBO Analysis}

NBO analysis is based on an approach of transformation of the multielectronic wave functions of the molecules in a localized form that corresponds to the elements with single center (single lone pair LP) and two centered orbitals (natural bonding $\sigma \pi$ and antiboning $\sigma * \pi *$ respectively). It provides an in-depth understanding of intra- and intermolecular orbital interactions in molecules between the NBOs of filled donors and the NBOs of empty acceptors [36]. For each donor NBO (i) and acceptor NBO (j), the stabilization energy associated with delocalization $\mathrm{i} \rightarrow \mathrm{j}$ can be estimated as follows:

$$
E^{(2)}=\Delta E_{i j}=q_{i} \frac{F(i, j)}{\varepsilon_{i} \varepsilon_{j}}
$$

Where qi is the electron density in the donor's orbital, F(i, j) is the non-diagonal element of the Fock matrix and $\varepsilon i$ and cj are the energies of the occupied orbitals $i$ and vacant $j$. By analyzing the interactions between the different acceptor and donor NBOs as well as the resulting stabilizing energy, clear information is obtained on the origin of the stabilization of a molecule. If the stabilization energy $\mathrm{E}^{(2)}$ associated with an interaction is high, the extent of stabilization will be greater.

\subsection{QTAIM Analysis}


Bader's Quantum Theory of Atoms in Molecules (QTAIM) is a useful tool for characterizing topological properties of chemical bonds [37]. The presence of chemical bonds between atoms and interatomic interactions is revealed by the presence of bond critical points (BCPs). The QTAIM method provides information on the electron density of a system that governs properties at BCPs. The QTAIM theory gives information on variations of electron density due to the formation of bonds or complexes [37]. Several parameters exist within the framework of the QTAIM theory, we have among others the electron density $\rho(r)$, its Laplacian $\nabla^{2} \rho(r)$, the potential local energy density $V(r)$, the local kinetic energy density $G(r)$ and the total kinetic energy density $H(r)$ at bond critical points (BCPs). In general, the parameter values of the QTAIM analysis at BCPs can describe the covalent or electrostatic nature of non-covalent interactions. According to Rozas et al [38] the interactions at the different BCPs can be classified as follows: for strong hydrogen bonds $\nabla^{2} \rho_{B C P} \prec 0, H_{B C P} \prec 0$; for medium hydrogen bonds $\nabla^{2} \rho_{B C P}>0, H_{B C P} \prec 0 ;$; for weak hydrogen bonds $\nabla^{2} \rho_{B C P}>0, H_{B C P}>0$. Also, the ratio $-\frac{G(r)}{V(r)}$ also allows a better understanding of the nature of the interaction at the different BCPs [39-41]. If $-\frac{G(r)}{V(r)}>1$ the bond is considered non-covalent. If $0,5 \prec-\frac{G(r)}{V(r)} \prec 1$ we have a partially covalent character of the bond. If $-\frac{G(r)}{V(r)} \prec 0,5$ we have a closed-shell interaction. Espinosa [42] proposed a relationship between the energy of the hydrogen bond $E_{H B}$ and the potential energy density $\left(\mathrm{V}_{\mathrm{BCP}}\right)$ at BCPs: $E_{H B}=\frac{1}{2}\left|V_{B C P}\right|$.

In addition, the ellipticity $\varepsilon$ to the different BCPs is defined as $\varepsilon=\frac{\lambda_{1}}{\lambda_{2}}-1: \lambda_{1}$ and $\lambda_{2}$ are eigenvalues of the Hessian of the electron density at BCP. This quantity estimates the extent to which the electron density is deformed in one direction relative to another. Ellipticity provides a measure of $\pi$ or $\sigma$ character of chemical bonds. A high value of ellipticity $(\varepsilon>0.1)$ indicates a $\pi$ character of the bond while a lower value reflects a $\sigma$ character of the bond [37]. Ellipticity reflects the stability of the bonds [39.43].

\subsection{NCI Analysis}

NCI (Non covalent interaction) analysis based on the correlation between the Reduced Gradient Density (RDG) and electron density $\rho(r)$ was recently developed by Yang et al $[44,45]$. The reduced gradient density is a fundamental dimensionless quantity of DFT, which is used to describe the deviation from a homogeneous distribution of electron density $[44,46]$. To some degree, the NCI analysis method can be considered as an extension of the AIM analysis [45]. The non-covalent interaction analysis method provides graphical visualization of molecule regions where noncovalent interactions occur and has demonstrated its ability to distinguish hydrogen bonds, van der Waals interactions and repulsive interactions [47]. Recently, the NCI-RDG analysis detected a low interaction compared to the AIM analysis [48]. To determine the presence of low interactions, $R D G=f\left(\operatorname{sign}\left(\lambda_{2}\right) \times \rho(r)\right)$ graphs were generated, where $\operatorname{sign}\left(\lambda_{2}\right) \times \rho(r)$ is the product of the electronic density $\rho(\mathrm{r})$ by the sign of the second eigenvalue of the hessian. The sign value $\operatorname{sign}\left(\lambda_{2}\right) \times \rho(r)$ is useful for predicting the nature of non-covalent interaction. For an attractive interaction $\operatorname{sign}\left(\lambda_{2}\right) \times \rho(r)<0$ (hydrogen bond) and for repulsive interactions $\operatorname{sign}\left(\lambda_{2}\right) \times \rho(r)>0$ (steric hindrance). For Van Der Walls interactions $\operatorname{sign}\left(\lambda_{2}\right) \times \rho(r)=0$.

\section{Results and Discussions}

\subsection{Study of Monomers}

The electronic properties obtained from QTAIM analysis, the geometrical parameters as well as the hyperconjugative interactions between the orbitals involved in the intramolecular hydrogen bonds of the M1M5 monomers are presented in Tables 1 and 2. The interactions $\mathrm{C}-\mathrm{H} \cdots \mathrm{N}, \mathrm{C}-\mathrm{H} \cdots \mathrm{H}-\mathrm{C}$ and $\mathrm{C}-\mathrm{H} \cdots \mathrm{Cl}$ were revealed by the QTAIM analysis (Figure 2). From the data in Table 1, it can be seen that the distances $d(H \cdots Y)$ and $d(X \cdots Y)$ are between 2.20881-2.27482 $\AA$ and 3.03051-3.05551 $\AA$ respectively. Also, the linearity angles $\angle X H Y$ are between $127.080-130.636^{\circ}$. These geometrical parameters indicate the presence of weak intramolecular hydrogen bond $[18,49,50]$. The $C-$ $H \cdots N$ bond have an electron density $\rho(r)$ in the range of 0.016617-0.018498 ea $a_{0}^{-3}$, a Laplacian $\nabla^{2} \rho(r)(0.052978$ $\left.0.058614 e a_{0}^{-5}\right)$ and a positive value of the total kinetic energy density $H(r)$ at different BCPs. These observations confirm the presence of weak intramolecular hydrogen bonds in M1-M5 monomers [51-54]. In addition, for all $C-H \cdots N$ bond, the ratio $-\frac{G(r)}{V(r)}>1$ implies that the $C-H \cdots N$ hydrogen bonds are non-covalent character [40, 41]. The interaction energies obtained for the $C-H \cdots N$ bonds are in the order of $3.3414375-3.8873625 \mathrm{kcal} / \mathrm{mol}$, these values confirm that these hydrogen bonds are weak [42]. Nevertheless, these interactions are stable because we have a low ellipticity value at the different BCPs.

The $C-H \cdots C l$ hydrogen bond is observed in the M4 molecule. At the Bcp of $\mathrm{C}-\mathrm{H} \cdots \mathrm{Cl}$ bond we have an electron density $\rho(r)=0,014863 e a_{0}^{-3}$, its Laplacian $\nabla^{2} \rho(r)=0,05587 e a_{0}^{-5}$ and a positive value of the total kinetic energy density $H(r)=0.002198 a u$. The $C-$ $H \cdots C l$ hydrogen bond is weak because $\rho(r)>0, \nabla^{2} \rho(r)>$ 0 and $H(r)>0$. In addition, the ratio $-\frac{G(r)}{V(r)}>1$ allows us to affirm that the $\mathrm{C}-\mathrm{H} \cdots \mathrm{Cl}$ interaction is non-covalent in nature. Except for the M4 monomer, $C-H \cdots H-$ $C$ interactions were observed in all monomers studied. At the Bcps of the different interactions $C-H \cdots H-C$ the electron density $\rho(r)$ varies between $0.008791-0.009004$ $e a_{0}^{-3}$ the Laplacian $\nabla^{2} \rho(r)$ is between 0.02-0.002492 au. 
Table 1. Topological parameters at bonds critical points of intramolecular interactions obtained by AIM analysis of M1-M5 monomers.

\begin{tabular}{|c|c|c|c|c|c|c|c|c|c|c|c|}
\hline & $\boldsymbol{X}-\boldsymbol{H} \cdots \boldsymbol{Y}$ & $\rho(r)_{X-H \cdots Y}$ & $\nabla^{2} \rho(r)_{X-H \cdots Y}$ & $\boldsymbol{V}(\boldsymbol{r})$ & $G(r)$ & $H(r)$ & $-\frac{G(r)}{V(r)}$ & $L(r)$ & $E_{H B}^{*}$ & $\Sigma E_{H B}$ & $\varepsilon(\boldsymbol{r})$ \\
\hline \multirow{2}{*}{ M1 } & $C-H \cdots N$ & 0.016346 & 0.052978 & -0.01065 & 0.011946 & 0.001298 & 1.122 & -0.01325 & 3.3414375 & \multirow{2}{*}{4.825475} & 0.015129 \\
\hline & $C-H \cdots H-C$ & 0.009004 & 0.038893 & -0.00473 & 0.007225 & 0.002499 & 1.529 & -0.00972 & 1.4840375 & & 1.235422 \\
\hline \multirow{2}{*}{ M2 } & $C-H \cdots N$ & 0.016715 & 0.05402 & -0.01094 & 0.01222 & 0.001284 & 1.117 & -0.01351 & 3.432425 & \multirow{2}{*}{4.8788125} & 0.014019 \\
\hline & $C-H \cdots H-C$ & 0.008813 & 0.038364 & -0.00461 & 0.007098 & 0.002492 & 1.541 & -0.00959 & 1.4463875 & & 1.434104 \\
\hline \multirow{2}{*}{ M3 } & $C-H \cdots N$ & 0.016757 & 0.054062 & -0.01096 & 0.012236 & 0.001279 & 1.117 & -0.01352 & 3.4387 & \multirow{2}{*}{4.8913625} & 0.014245 \\
\hline & $C-H \cdots H-C$ & 0.008791 & 0.039479 & -0.00463 & 0.007249 & 0.00262 & 1.566 & -0.00987 & 1.4526625 & & 3.146135 \\
\hline \multirow{2}{*}{ M4 } & $C-H \cdots N$ & 0.018498 & 0.058614 & -0.01239 & 0.013521 & 0.001133 & 1.091 & -0.01465 & 3.8873625 & \multirow{2}{*}{6.88995} & 0.001775 \\
\hline & $C-H \cdots C l$ & 0.014863 & 0.05587 & -0.00957 & 0.011769 & 0.002198 & 1.230 & -0.01396 & 3.0025875 & & 0.042812 \\
\hline \multirow{2}{*}{ M5 } & $C-H \cdots H-C$ & 0.008872 & 0.038579 & -0.00465 & 0.007146 & 0.002 & 1.5368 & -0.00965 & 1.4589375 & \multirow{2}{*}{4.8662625} & 1.370549 \\
\hline & $C-H \cdots N$ & 0.016617 & 0.053709 & -0.01086 & 0.012143 & 0.001 & 1.118 & -0.01343 & 3.407325 & & 0.014142 \\
\hline
\end{tabular}

Table 2. Geometrical parameters, hyperconjugative interactions, stabilization energy $E^{(2)}(k c a l / m o l)$ and charge transferred CT (me).

\begin{tabular}{|c|c|c|c|c|c|c|c|c|}
\hline & \multicolumn{5}{|c|}{ geometrical parameters } & \multicolumn{2}{|l|}{ NBO Interaction } & \multirow{2}{*}{ CT } \\
\hline & $X-H \cdots Y$ & $d(X-H)$ & $d(H \cdots Y)$ & $d(X \cdots Y)$ & $\angle X H Y$ & Transitions & $\boldsymbol{E}^{(2)}$ & \\
\hline M1 & $C-H \cdots N$ & 1.08660 & 2.27482 & 3.05551 & 127.080 & $n_{N 13}^{(1)} \rightarrow \sigma_{C 18-H 20}^{*}$ & 3.77 & CT \\
\hline M2 & $C-H \cdots N$ & 1.08654 & 2.26372 & 3.04660 & 127.239 & $n_{N 13}^{(1)} \rightarrow \sigma_{C 18-H 20}^{*}$ & 3.92 & 6.87 \\
\hline M3 & $C-H \cdots N$ & 1.08644 & 2.26282 & 3.04352 & 127.048 & $n_{N 13}^{(1)} \rightarrow \sigma_{C 18-H 20}^{*}$ & 3.97 & 7.144 \\
\hline \multirow{2}{*}{ M4 } & $C-H \cdots N$ & 1.08627 & 2.20881 & 3.03051 & 130.636 & $n_{N 13}^{(1)} \rightarrow \sigma_{C 18-H 20}^{*}$ & 4.91 & 7.144 \\
\hline & $C-H \cdots C l$ & 1.08127 & 2.50603 & 3.21292 & 122.021 & $n_{C l 30}^{(1)} \rightarrow \sigma_{C 17-H 19}^{*}$ & 2.57 & 9.096 \\
\hline M5 & $\mathrm{C}-\mathrm{H} \cdots \mathrm{N}$ & 1.08660 & 2.26653 & 3.04957 & 127.258 & $\mathrm{n}_{\mathrm{N} 13}^{(1)} \rightarrow \sigma_{\mathrm{C} 18-\mathrm{H} 20}^{*}$ & 3.90 & 5 \\
\hline
\end{tabular}

$C-H \cdots H-C$ interactions are weak and non-covalent in nature because $\rho(r)>0, \nabla^{2} \rho(r)>0, H(r)>0$ and $-\frac{G(r)}{V(r)}>$ 1 (Table 1). QTAIM parameters $\rho(r), \nabla^{2} \rho(r)$ and $E_{\text {int }}$ observed at the different Bcps of the $C-H \cdots H-C$ interactions are smaller than those of the $C-H \cdots N$ and $\mathrm{C}-\mathrm{H} \cdots \mathrm{Cl}$ interactions. The ellipticity values $\varepsilon(r)$ of the $C-H \cdots H-C$ interactions are large than those of the $\mathrm{C}-\mathrm{H} \cdots \mathrm{N}$ and $\mathrm{C}-\mathrm{H} \cdots \mathrm{Cl}$ interactions. As a result, $C-H \cdots H-C$ interactions are less stable and weak than $C-H \cdots N$ and $C-H \cdots C l$ interactions.

NBO analysis was performed on the M1-M5 monomers and the results obtained are shown in Table 2. NBO analysis revealed the presence of $\mathrm{C}-\mathrm{H} \cdots \mathrm{N}$ and $\mathrm{C}-\mathrm{H} \cdots \mathrm{Cl}$ interactions. Hyperconjugative interactions $n_{X} \rightarrow \sigma_{X-H}^{*}$ with stabilization energy ranging from $2.57-4.91 \mathrm{kcal} / \mathrm{mol}$ and corresponding transferred charges ranging from 5-9.096 me were obtained confirming the presence of weak intramolecular hydrogen bonds $\mathrm{C}-\mathrm{H} \cdots \mathrm{N}$ and $\mathrm{C}-\mathrm{H} \cdots \mathrm{Cl}$ [27].

\subsection{Dimer Studies}

\subsubsection{Stability, Geometrical Parameters and NBO Analysis of Complexes}

D2 dimer with an interaction energy of $-13.0604 \mathrm{kcal} / \mathrm{mol}$ is the most stable of the series. It is characterized by the presence of a hydrogen bond pair $N-H \cdots O$ (figure 3 ). The intermolecular distances $d(N \cdots O)$ and $d(H \cdots O)$ are in 2.87592 and $1.88814 \AA$ respectively. The $\angle N H O$ angle of the $N-H \cdots O$ bond is separated by $18.849^{\circ}$ from linearity. The variation in the length of the $N-H$ bond $\Delta d(N-H)>0$ implies an elongation its length during complexation. The vibrational stretching frequency $v_{N-H}$ of the monomer is $3646.9195 \mathrm{~cm}^{-1}$ while that of the dimer is $3435.3275 \mathrm{~cm}^{-1}$.
As a result, the variations in the vibrational stretch frequencies are negative. Based on the findings, we can say that the elongation of the $\mathrm{N}-\mathrm{H}$ bonds in the dimer is done in concert with a decrease in the elongation vibration frequencies of $\Delta v_{N-H}=-211.5919 \mathrm{~cm}^{-1}$ to the low frequencies (red shift). Thus in the D2 dimer the $N-$ $H \cdots O$ bonds are proper hydrogen bonds $[26,55]$. In Table 5 we have presented the non-covalent interactions observed in the dimers as well as the stabilization energy, the charge transferred and the variation of charges of the atoms $\mathrm{X}, \mathrm{H}$ and $\mathrm{Y}$ involved in the interactions $X-H \cdots Y$. The NBO analysis detected the presence of the $N-H \cdots O$ hydrogen bond characterized by the transfer of the lone pair of oxygen atom to the antibonding orbital $\sigma^{*}$ of the $N-H$ bond. Hyperconjugative interactions $n_{O 47}^{(1)} \rightarrow \sigma_{N 11-H 12}^{*}, n_{O 16}^{(1)} \rightarrow$ $\sigma_{N 42-H 43}^{*}$ on the one hand and $n_{O 47}^{(2)} \rightarrow \sigma_{N 11-H 12}^{*}, n_{O 16}^{(2)} \rightarrow$ $\sigma_{N 42-H 43}^{*}$ on the other hand with stabilization energies $E^{(2)}$ of 10.12 and $6.12 \mathrm{kcal} / \mathrm{mol}$ and charges transferred CT of 13.75 and 12.552 me respectively prove the presence of $N-H \cdots O$ hydrogen bonds [27].

The second stable complex in the series is the D5 dimer with an interaction energy $E_{\text {int }}=-11.94 \mathrm{kcal} / \mathrm{mol}$. This value is slightly lower than that of D2 dimer ( $E_{\text {int }}=-13.0604$ $\mathrm{kcal} / \mathrm{mol}$ ). The D5 dimer is characterized by the presence of a double $N-H \cdots O$ hydrogen bond (figure 3), an intermolecular $\mathrm{C}-\mathrm{H} \cdots \mathrm{O}$ hydrogen bond and intramolecular hydrogen bonds $C-H \cdots N$ and $C-$ $H \cdots H-C$. The $C-H \cdots O, C-H \cdots N$ and $C-H \cdots H-$ $C$ interactions (figure 4 ) were revealed by the AIM analysis of the complexes (see 3.2.3 section). the intermolecular distances $d(H \cdots Y)$ and $d(X \cdots Y)$ of the $N-H \cdots O$, $C-H \cdots O$ and $C-H \cdots N$ interactions are between 1.87984-3.18209 $\AA$ and 2.87331-3.00995 $\AA$ respectively. The length $d(H \cdots H)$ of the $C_{17}-H_{19} \cdots{ }_{17} H-C_{23}$ interaction is 
$2.16299 \AA$. The bonding angles of the intermolecular interactions $N-H \cdots O, C-H \cdots O$ and $C-H \cdots N$ are between $116.863-163.414^{\circ}$. The bonding angles of the $N-$ $H \cdots O$ interactions are closer to linearity than those of the $C-H \cdots O$ and $C-H \cdots N$ interactions. Also, we observe that the intermolecular distances $d(X \cdots Y)$ and $d(H \cdots Y)$ are smaller in $N-H \cdots O$ interactions. As a result, $N-H \cdots O$ interactions are stronger than $C-H \cdots O$ and $C-H \cdots N$ interactions. The $N-H$ bonds of the $N-H \cdots O$ interactions undergo an elongation $\Delta d(N-H)$ of 10.58 and $10.18 \mathrm{~m} \AA$ while the $C-H$ bonds of the $C-H \cdots O, C-H \cdots N$ and $C-H \cdots H-C$ interactions undergo a contraction in the range of $0.02-0.93 \mathrm{~m} \AA$. The elongation of the $N-H$ bond coincides with a shift of vibrational stretching frequency to low frequencies (red shift). Concerning the interactions $C-H \cdots O, C-H \cdots N$ and $C-H \cdots H-C$ a slight shift of the vibrational stretching frequency to high frequencies (blue shift) is observed (Table 4). These observations allow us to conclude that we have two types of non-covalent interactions in the D5 dimer; The $N-H \cdots O$ interaction characterized by an elongation of the $N-H$ bond $(\Delta d(N-H)>0)$ and a large red displacement of the vibrational stretching frequency is a proper hydrogen bond. The $C-H \cdots O, C-$ $H \cdots N$ and $C-H \cdots H-C$ interactions characterized by a blue shift of the vibrational stretching frequencies are improper hydrogen bonds $[26,55]$

The NBO analysis of D5 dimer and the NBO descriptors of hydrogen bond are recorded in Table 4. Except for the $\mathrm{C}_{17}-H_{19} \cdots_{27} \mathrm{H}-\mathrm{C}_{23}$ interaction, the NBO analysis detected the presence of observed hydrogen bonds.

$n_{O} \rightarrow \sigma_{N-H}^{*}$ interactions between the lone pair of hydrogen bond acceptor and the antibonding orbital $\sigma_{N-H}^{*}$ of hydrogen bond donor were revealed. The highest stabilization energy is obtained in the interaction $N_{42}-H_{43} \cdots O_{16}\left(n_{O 16}^{(1)} \rightarrow\right.$ $\left.\sigma_{N 42-H 43}^{*}\right)$ with an energy $E^{(2)}=11.06 \mathrm{kcal} / \mathrm{mol}$ and a charge transferred $\mathrm{CT}=12.637$ me followed by the interaction $N_{11}-H_{12} \cdots O_{47}\left(n_{O 47}^{(2)} \rightarrow \sigma_{N 11-H 12}^{*}\right)$ with a stabilization energy $E^{(2)}=9.28 \mathrm{kcal} / \mathrm{mol}$ and a charge transferred $C T=12.637 \mathrm{me}$. For improper hydrogen bonds, the NBO analysis revealed the presence $C_{18}-H_{20} \cdots N_{13}\left(n_{N 13}^{(1)} \rightarrow\right.$ $\left.\sigma_{C 18-H 20}^{*}, E^{(2)}=4.37 \mathrm{kcal} / \mathrm{mol} C T=7.993 \mathrm{me}\right)$ and $C-H \cdots O$ $\left(n_{O 47}^{(1)} \rightarrow \sigma_{C 2-H 7}^{*} E^{(2)}=0.05 \mathrm{kcal} / \mathrm{mol}, C T=0.07159 \mathrm{me}\right)$, however the stabilizing energy and charge transferred of these interactions are low compared to the $N_{11}-H_{12} \cdots O_{47}$ interaction.
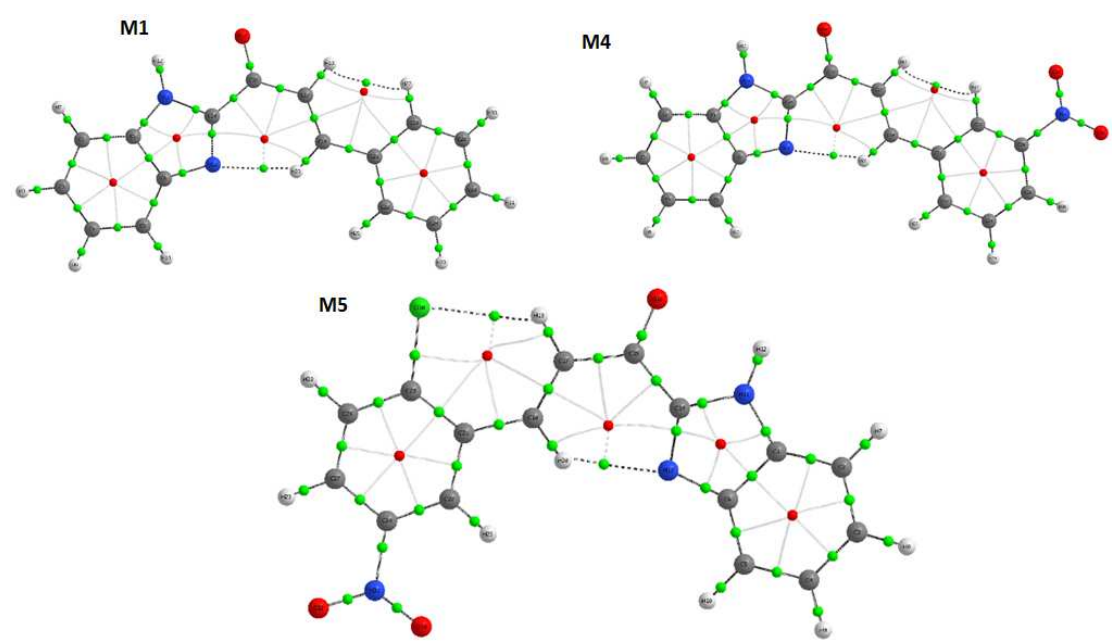

Figure 2. AIM molecular graphs of M1, M4 and M5 monomers at B3LYP 6-31+G (d, p) small green spheres are bonds critical points (Bcps).
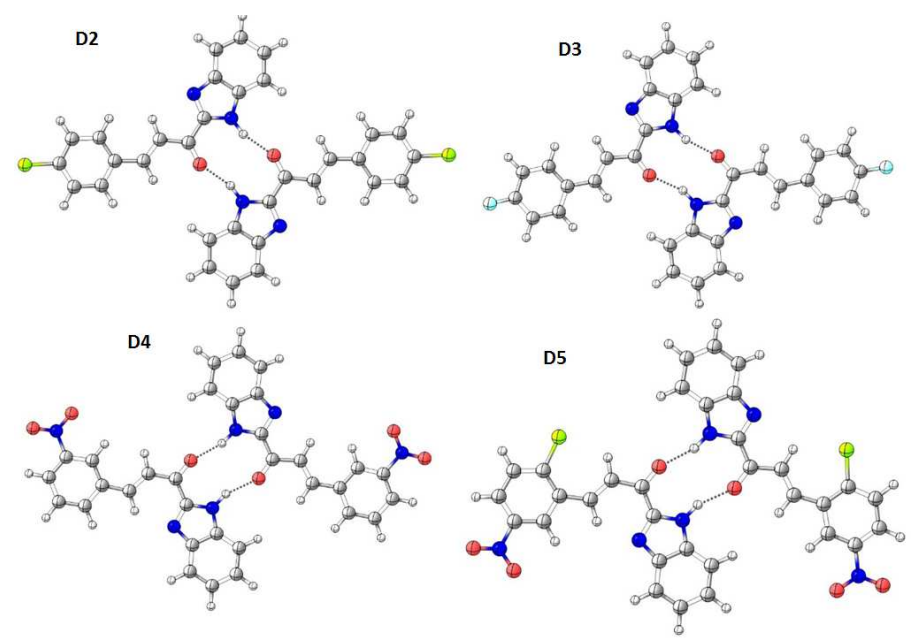

Figure 3. Optimized structures of D2-D5 dimers at B3LYP 6-31+G $(d, p)$. 
Table 3. Interaction energy $E_{\text {int }}(\mathrm{kcal} / \mathrm{mol})$ of D1-D5 dimers corrected by BSSE and ZPE.

\begin{tabular}{|c|c|c|c|c|c|c|}
\hline & $E_{\text {Dimer }}(\mathbf{a u})$ & $E_{\text {Monomer }}(\mathbf{a u})$ & $\triangle Z P E(\mathrm{au})$ & BSSE(au) & $E_{\text {int }}(\mathbf{a u})$ & $E_{\text {int }}$ \\
\hline D1 & -1603.45 & -801.715 & 0.001413 & 0.001237 & -0.016 & -10.0422 \\
\hline D2 & $-2522,64$ & -1261.31 & 0.001147 & 0.001287 & -0.02081 & -13.0604 \\
\hline D3 & -2012.47 & -1006.22 & 0.001394 & 0.001251 & -0.01846 & -11.581 \\
\hline D4 & -2931.64 & -1465.81 & 0.001296 & 0.001244 & -0.01879 & -11.7923 \\
\hline D5 & -1801.93 & -900.955 & 0.00127 & 0.001249 & -0.01904 & -11.9464 \\
\hline
\end{tabular}

Table 4. Geometrical parameters of $X-H \cdots Y, C-H \cdots H-C$ interactions (distances en $\AA$, angle $\angle X H Y$ (deg), change in bond length $\triangle d$ ( $m \AA \AA$ ) and stretching frequencies $v\left(\mathrm{~cm}^{-1}\right)$ and its shifts $\Delta v\left(\mathrm{~cm}^{-1}\right)$ for $C-H$ and $N-H$ bond.

\begin{tabular}{|c|c|c|c|c|c|c|c|c|}
\hline & $\begin{array}{l}X-H \cdots Y \\
C-H \cdots H-C\end{array}$ & $\begin{array}{l}d(H \cdots Y) \\
d(H \cdots H)\end{array}$ & $d(X \cdots Y)$ & $\angle X H Y$ & $\begin{array}{l}\Delta d(X-H) \\
\Delta d(C-H)\end{array}$ & $\begin{array}{l}v_{X-H}^{\text {monomer }} \\
v_{C-H}^{\text {monomer }}\end{array}$ & $\begin{array}{l}v_{X-H}^{\text {dimer }} \\
v_{C-H}^{\text {dimer }}\end{array}$ & $\begin{array}{l}\Delta v_{X-H} \\
\Delta v_{C-H}\end{array}$ \\
\hline \multirow{8}{*}{ D1 } & $N_{11}-H_{12} \cdots O_{47}$ & 1.88661 & 2.86706 & 159.887 & 10.19 & 3646.4497 & 3464.7658 & -181.6839 \\
\hline & $N_{42}-H_{43} \cdots O_{16}$ & 1.88650 & 2.86694 & 159.883 & 10.19 & 3646.4497 & 3464.7658 & -181.6839 \\
\hline & $C_{49}-H_{51} \cdots N_{44}$ & 2.23882 & 3.01365 & 126.449 & -0.32 & 3196.0143 & 3198.2511 & 2.2368 \\
\hline & $C-H_{58} \cdots H-C$ & 2.16137 & & & $\begin{array}{l}-0.02 \\
0.06\end{array}$ & 3180.628 & 3187.9229 & 7.2949 \\
\hline & $C_{18}-H_{20} \cdots N_{13}$ & 2.23854 & 3.01343 & 126.453 & -0.31 & 3196.0143 & 3198.2272 & 2.2129 \\
\hline & $C_{17}-H_{19} \cdots_{17} H-C_{23}$ & 2.16141 & & & $\begin{array}{l}0.02 \\
0.06\end{array}$ & 3180.628 & 3187.9156 & 7.2876 \\
\hline & $C_{33}-H_{38} \cdots O_{16}$ & 3.20049 & 3.80527 & 116.151 & -0.77 & 3206.6944 & 3216.294 & 9.5996 \\
\hline & $C_{2}-H_{7} \cdots O_{47}$ & 3.2006 & 3.80545 & 116.151 & -0.77 & 3206.6944 & 3216.2923 & 9.5979 \\
\hline \multirow{2}{*}{ D2 } & $N_{11}-H_{12} \cdots O_{47}$ & 1.88814 & 2.87592 & 161.532 & 11.18 & 3646.9194 & 3435.3275 & -211.5919 \\
\hline & $N_{42}-H_{43} \cdots O_{16}$ & 1.88814 & 2.87592 & 161.532 & 11.18 & 3646.9194 & 3435.3275 & -211.5919 \\
\hline \multirow{4}{*}{ D3 } & $N_{11}-H_{12} \cdots O_{49}$ & 1.91787 & 2.89576 & 159.212 & 10.58 & 3646.0336 & 3454.6697 & -191.3639 \\
\hline & $C_{2}-H_{7} \cdots O_{49}$ & 3.19843 & 3.81206 & 116.777 & -0.89 & 3198.1771 & 3199.25 & 1.0729 \\
\hline & $C_{18}-H_{20} \cdots N_{13}$ & 2.23393 & 3.00558 & 126.159 & 0.26 & 3197.4879 & 3194.7291 & -2.7588 \\
\hline & $C_{17}-H_{19} \cdots_{27} H-C_{23}$ & 2.16603 & & & $\begin{array}{l}-0.1 \\
-0.07\end{array}$ & 3193.0372 & 3245.2407 & 52.2035 \\
\hline \multirow{6}{*}{ D4 } & $N_{11}-H_{12} \cdots O_{49}$ & 1.87241 & 2.86785 & 163.983 & 10.26 & 3647.2604 & 3455.0778 & -192.1826 \\
\hline & $N_{44}-H_{45} \cdots O_{16}$ & 1.90681 & 2.88528 & 159.410 & 10.32 & 3647.2604 & 3455.0778 & -192.1826 \\
\hline & $C_{35}-H_{40} \cdots O_{16}$ & 3.19900 & 3.80798 & 116.457 & -0.97 & 3208.6194 & 3219.0874 & 10.468 \\
\hline & $C_{17}-H_{19} \cdots C l_{30}$ & 2.53155 & 3.20379 & 119.509 & -2.19 & 3258.3331 & 3288.7712 & 30.4381 \\
\hline & $C_{51}-H_{53} \cdots N_{46}$ & 2.17823 & 2.99290 & 129.873 & -0.21 & 3185.9744 & 3186.8562 & 0.8816 \\
\hline & $C_{50}-H_{52} \cdots C l_{63}$ & 2.50563 & 3.10349 & 122.398 & -0.07 & 3258.3331 & 3257.3667 & -0.9664 \\
\hline \multirow{4}{*}{ D5 } & $N_{11}-H_{12} \cdots O_{47}$ & 1.91387 & 2.88999 & 158.799 & 10.59 & 3646.7178 & 3451.4549 & -195.2629 \\
\hline & $N_{42}-H_{43} \cdots O_{16}$ & 1.87984 & 2.87331 & 163.414 & 10.18 & 3646.7178 & 3451.4549 & -195.2629 \\
\hline & $C_{2}-H_{7} \cdots O_{47}$ & 3.18209 & 3.79751 & 116.863 & -0.93 & 3214.7217 & 3217.4894 & 2.7677 \\
\hline & $C_{18}-H_{20} \cdots N_{13}$ & 2.23457 & 3.00995 & 126.481 & -0.31 & 3196.3516 & 3197.94 & 1.5884 \\
\hline
\end{tabular}

$\Delta v=v_{X-H}^{\text {dimer }}-v_{X-H}^{\text {monomer }}, \Delta d=d_{X-H}^{\text {dimer }}-d_{X-H}^{\text {monomer }}$

Table 5. Hyperconjugative interactions, stabilization energies $E^{(2)}(k c a l / m o l)$ and charge transferred CT (me) as well as the variation of the charges of atoms $X$, $Y$ and $H$ with dimerization.

\begin{tabular}{|c|c|c|c|c|c|c|c|c|}
\hline Dimers & Contact & Interaction & $E^{(2)}$ & $\Sigma E^{(2)}$ & CT & $\Delta q_{X}$ & $\Delta q_{H}$ & $\Delta q_{Y}$ \\
\hline \multirow{9}{*}{$\mathbf{D}_{1}$} & $N_{11}-H_{12} \cdots O_{47}$ & $n_{O 47}^{(1)} \rightarrow \sigma_{N 11-H 12}^{*}$ & 10.41 & \multirow{8}{*}{40.66} & 14.32 & 0.0539 & -0.0152 & 0.0122 \\
\hline & $N_{11}-H_{12} \cdots O_{47}$ & $n_{O 47}^{(2)} \rightarrow \sigma_{N 11-H 12}^{*}$ & 5.57 & & 11.74 & 0.0539 & -0.0152 & 0.0122 \\
\hline & $N_{42}-H_{43} \cdots O_{16}$ & $n_{O 16}^{(1)} \rightarrow \sigma_{N 42-H 43}^{*}$ & 10.41 & & 14.32 & 0.0539 & -0.0152 & 0.0122 \\
\hline & $N_{42}-H_{43} \cdots O_{16}$ & $n_{O 16}^{(2)} \rightarrow \sigma_{N 42-H 43}^{*}$ & 5.57 & & 11.74 & 0.0539 & -0.0152 & 0.0122 \\
\hline & $C_{2}-H_{7} \cdots O_{47}$ & $n_{O 47}^{(2)} \rightarrow \sigma_{C 2-H 7}^{*}$ & 0.06 & & 0.11834 & 0.03216 & -0.01012 & 0.0539 \\
\hline & $C_{33}-H_{38} \cdots O_{16}$ & $n_{O 16}^{(2)} \rightarrow \sigma_{C 33-H 38}^{*}$ & 0.06 & & 0.11834 & 0.03203 & -0.01012 & 0.0539 \\
\hline & $C_{49}-H_{51} \cdots N_{44}$ & $n_{N 44}^{(1)} \rightarrow \sigma_{C 49-H 51}^{*}$ & 4.29 & & 7.705 & -0.01551 & -0.00185 & 0.00543 \\
\hline & $C_{18}-H_{20} \cdots N_{13}$ & $n_{N 13}^{(1)} \rightarrow \sigma_{C 18-H 20}^{*}$ & 4.29 & & 7.705 & -0.01551 & -0.00185 & 0.00543 \\
\hline & $N_{11}-H_{12} \cdots O_{47}$ & $n_{O 47}^{(1)} \rightarrow \sigma_{N 11-H 12}^{*}$ & 10.12 & & 13.7468 & 0.06631 & -0.0153 & 0.00029 \\
\hline \multirow[t]{2}{*}{ D2 } & $N_{11}-H_{12} \cdots O_{47}$ & $n_{O 47}^{(1)} \rightarrow \sigma_{N 11-H 12}^{*}$ & 6.12 & 32.48 & 12.552 & 0.06631 & -0.0153 & 0.00029 \\
\hline & $N_{42}-H_{43} \cdots O_{16}$ & $n_{O 16}^{(1)} \rightarrow \sigma_{N 42-H 43}^{*}$ & 10.12 & & 13.7468 & 0.06631 & -0.0153 & 0.00029 \\
\hline
\end{tabular}




\begin{tabular}{|c|c|c|c|c|c|c|c|c|}
\hline Dimers & Contact & Interaction & $E^{(2)}$ & $\Sigma E^{(2)}$ & CT & $\Delta q_{X}$ & $\Delta q_{H}$ & $\Delta q_{Y}$ \\
\hline \multirow{7}{*}{ D3 } & $N_{42}-H_{43} \cdots O_{16}$ & $n_{O 16}^{(2)} \rightarrow \sigma_{N 42-H 43}^{*}$ & 6.12 & \multirow{6}{*}{44.24} & 12.552 & 0.06631 & -0.0153 & 0.00029 \\
\hline & $N_{44}-H_{45} \cdots O_{16}$ & $n_{016}^{(1)} \rightarrow \sigma_{N 44-H 45}^{*}$ & 13.14 & & 17.6975 & 0.00076 & -0.01588 & 0.06656 \\
\hline & $N_{44}-H_{45} \cdots O_{16}$ & $n_{O 16}^{(2)} \rightarrow \sigma_{N 44-H 45}^{*}$ & 6.82 & & 13.9593 & 0.00076 & -0.01588 & 0.06656 \\
\hline & $N_{11}-H_{12} \cdots O_{49}$ & $n_{O 49}^{(1)} \rightarrow \sigma_{N 11-H 12}^{*}$ & 11.27 & & 15.5 & 0.01394 & -0.01758 & 0.08213 \\
\hline & $N_{11}-H_{12} \cdots O_{49}$ & $n_{O 49}^{(2)} \rightarrow \sigma_{N 11-H 12}^{*}$ & 7.96 & & 16.11 & 0.01394 & -0.01758 & 0.08213 \\
\hline & $C_{2}-H_{7} \cdots O_{49}$ & 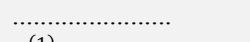 & & & & & & \\
\hline & $C_{18}-H_{20} \cdots N_{13}$ & $n_{N 13}^{(1)} \rightarrow \sigma_{C 18-H 20}^{*}$ & 5.05 & \multirow{10}{*}{43.51} & 9.198 & -0.00788 & -0.00523 & 0.00023 \\
\hline \multirow{10}{*}{ D4 } & $N_{11}-H_{12} \cdots O_{49}$ & $n_{O 49}^{(1)} \rightarrow \sigma_{N 11-H 12}^{*}$ & 11.31 & & 15.5 & 0.01552 & -0.01386 & 0.059595 \\
\hline & $N_{11}-H_{12} \cdots O_{49}$ & $n_{O 49}^{(2)} \rightarrow \sigma_{N 11-H 12}^{*}$ & 5.17 & & 10.68 & 0.01552 & -0.01386 & 0.059595 \\
\hline & $N_{44}-H_{45} \cdots O_{16}$ & $n_{O 16}^{(1)} \rightarrow \sigma_{N 44-H 45}^{*}$ & 9.57 & & 13.185 & 0.01581 & -0.01437 & 0.06804 \\
\hline & $N_{44}-H_{45} \cdots O_{16}$ & $n_{O 16}^{(2)} \rightarrow \sigma_{N 44-H 45}^{*}$ & 5.61 & & 11.35 & 0.01581 & -0.01437 & 0.06804 \\
\hline & $C_{17}-H_{19} \cdots C l_{30}$ & $n_{C l 30}^{(1)} \rightarrow \sigma_{C 17-H 19}^{*}$ & 0.67 & & 0.756 & 0.00392 & -0.01466 & 0.00386 \\
\hline & & $n_{C l 30}^{(2)} \rightarrow \sigma_{C 17-H 19}^{*}$ & 2.35 & & 4.4157 & & & \\
\hline & $C_{50}-H_{52} \cdots C l_{63}$ & $n_{C l 63}^{(1)} \rightarrow \sigma_{C 50-H 52}^{*}$ & 0.73 & & 0.834 & 0.00382 & 0.00325 & 0.00264 \\
\hline & & $n_{C l 63}^{(2)} \rightarrow \sigma_{C 50-H 52}^{*}$ & 2.65 & & 5.246 & & & \\
\hline & $C_{51}-H_{53} \cdots N_{46}$ & $n_{N 46}^{(1)} \rightarrow \sigma_{C 51-H 53}^{*}$ & 5.51 & & 10.394 & 0.00455 & -0.00181 & -0.02136 \\
\hline & $C_{35}-H_{40} \cdots O_{16}$ & 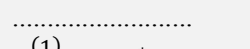 & & & & & & \\
\hline \multirow{6}{*}{ D5 } & $N_{11}-H_{12} \cdots O_{47}$ & $n_{O 47}^{(1)} \rightarrow \sigma_{N 11-H 12}^{*}$ & 9.28 & \multirow{6}{*}{35.57} & 12.637 & 0.01242 & -0.01558 & 0.06314 \\
\hline & $N_{11}-H_{12} \cdots O_{47}$ & $n_{O 47}^{(2)} \rightarrow \sigma_{N 11-H 12}^{*}$ & 5.71 & & 12.05 & 0.01242 & -0.01558 & 0.06314 \\
\hline & $N_{42}-H_{43} \cdots O_{16}$ & $n_{O 16}^{(1)} \rightarrow \sigma_{N 42-H 43}^{*}$ & 11.06 & & 15.2 & 0.01831 & -0.01482 & 0.05552 \\
\hline & $N_{42}-H_{43} \cdots O_{16}$ & $n_{O 16}^{(2)} \rightarrow \sigma_{N 42-H 43}^{*}$ & 5.10 & & 10.58 & 0.01831 & -0.01482 & 0.05552 \\
\hline & $\mathrm{C}_{2}-\mathrm{H}_{7} \cdots \mathrm{O}_{47}$ & $n_{O 47}^{(1)} \rightarrow \sigma_{C 2-H 7}^{*}$ & 0.05 & & 0.07159 & 0.03285 & -0.00649 & 0.06314 \\
\hline & $C_{18}-H_{20} \cdots N_{13}$ & $n_{N 13}^{(1)} \rightarrow \sigma_{C 18-H 20}^{*}$ & 4.37 & & 7.993 & -0.01647 & 0.0018 & 0.00395 \\
\hline
\end{tabular}

D4 Dimer is the third most stable complex in the series. It has an interaction energy $E_{\text {int }}=-11.7933 \mathrm{kcal} / \mathrm{mol}$. This dimer is formed by a double $N-H \cdots O$ hydrogen bond (figure 3). In addition, the QTAIM analysis detected the presence of $\mathrm{C}-\mathrm{H} \cdots \mathrm{O}, \mathrm{C}-\mathrm{H} \cdots \mathrm{Cl}$ and $\mathrm{C}-\mathrm{H} \cdots \mathrm{N}$ interactions (figure 4) (see 3.2.3 section). The intermolecular distances $d(X \cdots Y), d(H \cdots Y)$ of the interactions observed in the D4 dimer range from 1.87241-3.19900 $\AA$ and 2.86785$3.80798 \AA$ respectively. Regarding the linearity angles, they vary between $116.457-163.983^{\circ}$. The distances of the intermolecular interactions $N-H \cdots O$ are generally smaller than those of the intramolecular interactions $\mathrm{C}-\mathrm{H} \cdots \mathrm{Cl}$ and $C-H \cdots N$ and the bonding angles $\angle N H O$ are closer to linearity. The $N-H \cdots O$ intermolecular interaction are consequently stronger than the $\mathrm{C}-\mathrm{H} \cdots \mathrm{Cl}$ and $\mathrm{C}-\mathrm{H} \cdots \mathrm{N}$ intramolecular interaction. With complexation, we observe an elongation of the $N-H$ bonds of the $N-H \cdots O$ interactions $(\Delta d(N-H)>0)$ while in the $C-H \cdots O$, $\mathrm{C}-\mathrm{H} \cdots \mathrm{Cl}$ and $\mathrm{C}-\mathrm{H} \cdots \mathrm{N}$ interactions we observe a shortening of the $C-H$ bonds $(\Delta d(N-H)<0)$. The elongation of the $N-H$ bonds is combined with a red shift of the vibrational stretching frequency $\Delta v_{N-H}=-192.1826$ $\mathrm{cm}^{-1}$ and the contraction of the $C-H$ bonds of the $C-$ $H \cdots O, C-H \cdots C l$ and $C-H \cdots N$ interactions is combined with a small blue shift of the vibrational stretching frequencies $\Delta v_{C-H}$ which is comprised between 0.8816 $30.4381 \mathrm{~cm}^{-1}$. It can be seen that the blue shifts of the vibrational stretching frequencies of the $C-H$ bonds are small compared to the shifts of the $N-H$ bonds. This low blue shift of the $C-H$ bonds implies that the $C-H \cdots O$, $C-H \cdots C l$ and $C-H \cdots N$ interactions are weaker than the $N-H \cdots O$ interactions. However, the contraction of the $\mathrm{C}-\mathrm{H}$ bond in the $\mathrm{C}_{50}-\mathrm{H}_{52} \cdots \mathrm{Cl}_{63}$ interaction is associated with a low red shift of the stretch vibration frequency
$\Delta v_{C-H}=-0.9664 \mathrm{~cm}^{-1}$. Consequently, $N-H \cdots O$ interactions are proper hydrogen bonds and $C-H \cdots O$, $\mathrm{C}-\mathrm{H} \cdots \mathrm{Cl}$ and $\mathrm{C}-\mathrm{H} \cdots \mathrm{N}$ interactions are improper hydrogen bonds $[26,55]$.

The elongation of the $X-H$ bond in the $X-H \cdots Y$ interaction is generally attributed to the charge transferred of the interaction $n_{Y} \rightarrow \sigma_{X-H}^{*}$ between the lone pair of the proton acceptor and the anti-bonding orbital $\sigma_{X-H}^{*}$ of the donor [24]. The consequence of these charges transferred are measured by the second-order stabilization energy $E^{(2)}$ obtained by the NBO analysis presented in Table 5. We observe that the largest stabilization energies $E^{(2)}$ and charge transferred are obtained for the interaction $N_{11}-H_{12} \cdots O_{49}$ $\left(n_{O 49}^{(1)} \rightarrow \sigma_{N 11-H 12}^{*}, E^{(2)}=11.31 \mathrm{kcal} / \mathrm{mol}, C T=15.52 \mathrm{e}\right)$ followed by $N_{44}-H_{45} \cdots O_{16}$ interaction $\left(n_{O 16}^{(1)} \rightarrow \sigma_{N 44-H 45}^{*}\right.$, $\left.E^{(2)}=9.57 \mathrm{kcal} / \mathrm{mol}, C T=12.637 \mathrm{e}\right)$. The total stabilization energy $\Sigma E^{(2)}$ in the dimer is $31.66 \mathrm{kcal} / \mathrm{mol}$ which implies a large charge transfer in the D4 dimer through $N-H \cdots O$, $\mathrm{C}-\mathrm{H} \cdots \mathrm{O}, \mathrm{C}-\mathrm{H} \cdots \mathrm{Cl}$ and $\mathrm{C}-\mathrm{H} \cdots \mathrm{N}$ interactions.

With an interaction energy of $-11.581 \mathrm{kcal} / \mathrm{mol}$ the D3 dimer is the fourth stable complex studied. It is also characterized by a double $N-H \cdots O$ interaction (figure 3) but also by the $\mathrm{C}-\mathrm{H} \cdots \mathrm{O}, \mathrm{C}-\mathrm{H} \cdots \mathrm{Cl}$ and $\mathrm{C}-\mathrm{H} \cdots \mathrm{H}-\mathrm{C}$ interactions (figure 4). revealed by the QTAIM analysis (see 3.2.3 section). The intermolecular distances of the $N-$ $H \cdots O$ interactions $(d(H \cdots O)=1.87241-1.90681$ $\AA ; d(N \cdots O)=3.00558-3.81206 \AA$ ) (Table 4) are lower than that of $C-H \cdots O$ and $C-H \cdots N$ interactions $(d(H \cdots Y)=$ 2.23393-3.19843 $\AA ; d(X \cdots Y)=3.00558-3.81206 \AA)$. The intermolecular distance $d(H \cdots H)$ of the $C-H \cdots H-C$ interaction is $2.16603 \AA$. the $\angle N H O$ bonding angles of the $N-H \cdots O$ interactions $\left(159.410-163.983^{\circ}\right)$ are closer to linearity than those of the $C-H \cdots O$ and $C-H \cdots N$ 
interactions $\left(116.777-126.159^{\circ}\right)$. In addition, the analysis of the bond lengths shows that the $N-H$ and $C-H$ bonds of the $N_{11}-H_{12} \cdots O_{49}, N_{44}-H_{45} \cdots O_{16}$ and $C_{18}-H_{20} \cdots N_{13}$ interactions undergo elongation $(\Delta d(C-H)>$ $0, \Delta d(N-H)>0)$ while that of the $C_{2}-H_{7} \cdots O_{49}$ interaction undergoes contraction with complexation. This variation in bond length is combined with a red shift of vibrational stretching frequency of the $N-H$ and $C-H$ bonds of the $N_{11}-H_{12} \cdots O_{49}, N_{44}-H_{45} \cdots O_{16}$ and $C_{18}-H_{20} \cdots N_{13}$ interactions. However, a blue shift of vibrational stretching frequency of the $\mathrm{C}-\mathrm{H}$ bond is observed in the $\mathrm{C}_{2}-H_{7} \cdots \mathrm{O}_{49}$ and $\mathrm{C}_{17}-\mathrm{H}_{19} \cdots_{17} \mathrm{H}-\mathrm{C}_{23}$ interactions. With these observations we can affirm that the interactions $N-H \cdots O$ and $C-H \cdots N$ are proper hydrogen bonds and the interactions $C-H \cdots O$ and $C-H \cdots H-C$ are improper hydrogen bonds $[26,55]$.

The NBO analysis was performed and the stabilization energy due to hyperconjugative interactions $n_{Y} \rightarrow \sigma_{X-H}^{*}$ of the $X-H \cdots Y$ interactions are recorded in Table 5. This analysis clearly shows the presence of hydrogen bonds $N_{44}-H_{45} \cdots O_{16} \quad\left(n_{O 16}^{(1)} \rightarrow \sigma_{N 44-H 45}^{*}, \Delta E^{(2)}=13,14 \mathrm{kcal} /\right.$ $\left.\mathrm{mol} ; n_{O 16}^{(2)} \rightarrow \sigma_{N 44-H 45}^{*}, \Delta E^{(2)}=6,82 \mathrm{kcal} / \mathrm{mol}\right), N_{11}-$ $H_{12} \cdots O_{49} \quad\left(n_{O 49}^{(1)} \rightarrow \sigma_{N 11-H 12}^{*}, E^{(2)}=11.27 \mathrm{kcal} / \mathrm{mol}\right.$; $\left.n_{O 49}^{(2)} \rightarrow \sigma_{N 11-H 12}^{*}, \Delta E^{(2)}=7.96 \mathrm{kcal} / \mathrm{mol}\right)$ and $C_{18}-$ $H_{20} \cdots N_{13}\left(n_{N 13}^{(1)} \rightarrow \sigma_{C 18-H 20}^{*}, E^{(2)}=5.05 \mathrm{kcal} / \mathrm{mol}\right)$ [27]. These observed interactions due to the overlap between the free doublet of the atom $n_{Y}$ and the antibonding orbital $\sigma_{X-H}^{*}$ have as total stabilization energies $\Sigma E^{(2)}$ equal to 44.24 $\mathrm{kcal} / \mathrm{mol}$ for the $N_{44}-H_{45} \cdots O_{16}, N_{11}-H_{12} \cdots O_{49}$ and $C_{18}-H_{20} \cdots N_{13}$ interactions, therefore there is a strong charge transfer in the D3 dimer which contributes to its stability.

The least stable complex is D1 dimer. It has an interaction energy $E_{\text {int }}=-10.0422 \mathrm{kcal} / \mathrm{mol}$ significantly lower than that of D2-D5 dimers. It is stabilized by a $N-H \cdots O$ hydrogen bond (figure 3). and the $C-H \cdots N, C-H \cdots O$ and $C-H \cdots H-C$ hydrogen bond revealed by the AIM analysis (figure 4). The intermolecular distances $d(H \cdots Y), d(X \cdots Y)$ are between 1.88661-3.20049 $\AA$ and 2.86694-3.80545 $\AA$ respectively. The corresponding linearity angles $\angle X H Y$ vary between $116.151-159.887^{\circ}$. During complexation, the N-H bonds of the $N-H \cdots O$ interactions lengthen $(\Delta d(N-H)>0)$ with a red shift of their vibrational stretching frequency of $\Delta v_{N-H}=-181.6839 \mathrm{~cm}^{-1}$ while the $\mathrm{C}-\mathrm{H}$ bonds of the $\mathrm{C}-H \cdots N$ and $C-H \cdots O$ interactions shorten with a blue shift $\Delta v_{C-H}$ (between 2.2129-9.5996 $\mathrm{cm}^{-1}$ ) of their vibrational stretching frequencies. $N-H \cdots O$ interactions are therefore stronger than $\mathrm{C}-\mathrm{H} \cdots \mathrm{O}, \mathrm{C}-\mathrm{H} \cdots \mathrm{N}$ and $\mathrm{C}-\mathrm{H} \cdots H-\mathrm{C}$ interactions. Consequently, the $N-H \cdots O$ interactions are proper hydrogen bonds and the $C-H \cdots O, C-H \cdots N$ and $C-H \cdots H-C$ interactions are improper hydrogen bonds [26, 55]. The hyperconjugative interactions $n_{X} \rightarrow$ $\sigma_{X-H}^{*}$ obtained by the NBO analysis allow to confirm the presence of the $N-H \cdots O, C-H \cdots O$ and $C-H \cdots N$ hydrogen bonds in the D1 dimer [27]. The corresponding stabilization energies vary between $0.06-10.41 \mathrm{kcal} / \mathrm{mol}$. The total stabilization energy $\Sigma E^{(2)}$ is $40.66 \mathrm{kcal} / \mathrm{mol}$, confirming the presence of a high charge transfer through the $N-$ $H \cdots O, C-H \cdots O$ and $C-H \cdots N$ hydrogen bonds.

The formation of the $X-H \cdots Y$ hydrogen bond is related to the increase of the positive charge of the $\mathrm{H}$ atom and the negative charge of the $X$ and $Y$ atoms. The change in atomic charges resulting from the complexing of the D1-D5 dimers is recorded in Table 5. Apart from $\mathrm{C}_{50}-\mathrm{H}_{52} \cdots \mathrm{Cl}_{63}$ (D4) and $C_{18}-H_{20} \cdots N_{13}\left(\mathrm{D}_{5}\right)$ hydrogen bonds, the atomic charges of hydrogen in the $X-H \cdots Y$ interactions of the D1D5 dimers increase $\left(\Delta q_{H} \prec 0\right)$ while that of the $\mathrm{N}, \mathrm{O}$ and $\mathrm{C}$ atoms decrease $\left(\Delta q_{O}>0, \Delta q_{C}>0, \Delta q_{N}>0\right) \quad$ with complexation. Nevertheless, in the intramolecular bonds of the D1 and D4 dimers, the increase in the positive charge of the hydrogen atom $\mathrm{H}$ is combined with an increase of the charge of the nitrogen atom. In the interactions $X-H \cdots Y$ of the D1-D5 dimers, the increase of positive charge of the hydrogen atom $\mathrm{H}$ and that of the negative charge of $\mathrm{X}$ atom observed is a signature of the hydrogen bond $[27,56]$. The lone pairs of the $\mathrm{Y}$ atoms $(\mathrm{Y} \equiv \mathrm{O}, \mathrm{N}, \mathrm{Cl})$ involved in hyperconjugative interactions $n_{Y} \rightarrow \sigma_{X-H}^{*}$ are hybridized $s p^{m}(m \in 0.74-99.99)$ with electron densities ranging from 1.90394 to $1.99144 \mathrm{e}$. These electron densities less than 2 show that these lone pairs are delocalized, particularly in the antibonding orbital $\sigma^{*}$, which have an electron density between $0.01378 \mathrm{e}$ and $0.04825 \mathrm{e}$. the electron densities of the antibonding orbitals of the N-H, C-H bonds and the S character of the nitrogen and carbon atoms $((N)-H$, $(C)-H)($ Table 7$)$ increase with complexity. this increase in the $\mathrm{S}$ character of nitrogen and carbon atoms indicates a rehybridization of the orbitals of the latter during the formation of the hydrogen bonds $\mathrm{N}-\mathrm{H} \cdots \mathrm{O}, \mathrm{C}-\mathrm{H} \cdots \mathrm{O}$ and $C-H \cdots N$ [27].

Table 6. Lone pairs $\left(n_{Y}\right)$ and antibonding orbitals $\left(\sigma_{X-H}^{*}\right)$ hybridization states, their respective electronic densities as well as the variation of the electronic density of the antibonding orbitals $\sigma_{X-H}^{*}$ upon complexation.

\begin{tabular}{|c|c|c|c|c|c|c|c|c|}
\hline \multirow{3}{*}{ dimers } & \multirow{3}{*}{ Contact } & \multirow{2}{*}{\multicolumn{2}{|c|}{$\begin{array}{l}\text { Donor } \\
n_{Y} \\
\end{array}$}} & \multicolumn{5}{|l|}{ Acceptor } \\
\hline & & & & \multicolumn{5}{|l|}{$\sigma_{X-H}^{*}$} \\
\hline & & Hybridization & ED & Hybridization & ED & $\Delta \sigma^{*}$ & $E D_{X}(\%)$ & $E D_{H}(\%)$ \\
\hline \multirow{4}{*}{$\mathrm{D}_{1}$} & $\begin{array}{l}N_{11}-H_{12} \cdots O_{47} \\
N_{11}-H_{12} \cdots O_{47}\end{array}$ & $\begin{array}{l}s p^{0.74} \\
s p^{47.79}\end{array}$ & $\begin{array}{l}1.96219 \\
1.90394\end{array}$ & $\sigma_{N 11-H 12}^{*}=0.4892\left(s p^{2.01}\right)_{N}-0.8722(s)_{H}$ & 0.04462 & 0.02385 & 23.93 & 76.07 \\
\hline & $\begin{array}{l}N_{42}-H_{43} \cdots O_{16} \\
N_{42}-H_{43} \cdots O_{16}\end{array}$ & $\begin{array}{l}s p^{0.74} \\
s p^{47.79}\end{array}$ & $\begin{array}{l}1.96219 \\
1.90394\end{array}$ & $\sigma_{N 42-H 43}^{*}=0.4892\left(s p^{2.01}\right)_{N}-0.8722(s)_{H}$ & 0.04462 & 0.02385 & 23.93 & 76.07 \\
\hline & $C_{18}-H_{20} \cdots N_{13}$ & $s p^{1.97}$ & 1.92099 & $\sigma_{C_{18}-H_{20}}^{*}=0.5999\left(s p^{1.97}\right)_{C}-0.8001(s)_{H}$ & 0.02305 & 0.00125 & 35.99 & 64.01 \\
\hline & $C_{2}-H_{7} \cdots O_{47}$ & $s p^{0.47}$ & 1.96219 & $\sigma_{C_{2}-H_{7}}^{*}=0.6070\left(s p^{2.23}\right)_{C}-0.7947(s)_{H}$ & 0.01378 & 0.00007 & 36.85 & 63.15 \\
\hline
\end{tabular}




\begin{tabular}{|c|c|c|c|c|c|c|c|c|}
\hline \multirow{3}{*}{ dimers } & \multirow{3}{*}{ Contact } & \multicolumn{2}{|l|}{ Donor } & \multicolumn{5}{|l|}{ Acceptor } \\
\hline & & \multicolumn{2}{|l|}{$n_{Y}$} & \multicolumn{5}{|l|}{$\sigma_{X-H}^{*}$} \\
\hline & & Hybridization & ED & Hybridization & ED & $\Delta \sigma^{*}$ & $E D_{X}(\%)$ & $E D_{H}(\%)$ \\
\hline \multirow{4}{*}{$\mathrm{D}_{2}$} & $N_{11}-H_{12} \cdots O_{47}$ & $s p^{0.74}$ & 1.96227 & \multirow{2}{*}{$\sigma_{N 11-H 12}^{*}=0.4893\left(s p^{2.03}\right)_{N}-0.8721(s)_{H}$} & \multirow{2}{*}{0.04516} & \multirow{2}{*}{0.02462} & \multirow{2}{*}{23.94} & \multirow{2}{*}{76.06} \\
\hline & $N_{11}-H_{12} \cdots O_{47}$ & $s p^{55.38}$ & 1.89373 & & & & & \\
\hline & $N_{42}-H_{43} \cdots O_{16}$ & $s p^{0.74}$ & 1.96227 & \multirow{2}{*}{$\sigma_{N 42-H 43}^{*}=0.4893\left(s p^{2.02}\right)_{N}-0.8721(s)_{H}$} & \multirow{2}{*}{0.04516} & \multirow{2}{*}{0.02462} & \multirow{2}{*}{23.94} & \multirow{2}{*}{76.06} \\
\hline & $N_{42}-H_{43} \cdots O_{16}$ & $s p^{55.38}$ & 1.89373 & & & & & \\
\hline \multirow{5}{*}{$\mathrm{D}_{3}$} & $N_{44}-H_{45} \cdots O_{16}$ & $s p^{0.71}$ & 1.95863 & \multirow{2}{*}{$\sigma_{N 44-H 45}^{*}=0.6165\left(s p^{2.02}\right)_{N}-0.7873(s)_{H}$} & \multirow{2}{*}{0.04746} & \multirow{2}{*}{0.02721} & \multirow{2}{*}{23.79} & \multirow{2}{*}{76.37} \\
\hline & $N_{44}-H_{45} \cdots O_{16}$ & $s p^{28.54}$ & 1.91131 & & & & & \\
\hline & $N_{11}-H_{12} \cdots O_{49}$ & $s p^{0.70}$ & 1.96083 & \multirow{2}{*}{$\sigma_{N 11-H 12}^{*}=0.4861\left(s p^{1.97}\right)_{N}-0.8739(s)_{H}$} & \multirow{2}{*}{0.04825} & \multirow{2}{*}{0.028} & \multirow{2}{*}{23.63} & \multirow{2}{*}{76.37} \\
\hline & $N_{11}-H_{12} \cdots O_{49}$ & $s p^{33.83}$ & 1.90013 & & & & & \\
\hline & $C_{18}-H_{20} \cdots N_{13}$ & $s p^{1.88}$ & 1.92310 & $\sigma_{C 18-H 20}^{*}=0.5973\left(s p^{2.93}\right)_{C}-0.8020(s)_{H}$ & 0.02438 & 0.00205 & 35.68 & 63.32 \\
\hline \multirow{9}{*}{$\mathrm{D}_{4}$} & $N_{11}-H_{12} \cdots O_{49}$ & $s p^{0.74}$ & 1.96322 & \multirow{2}{*}{$\sigma_{N 11-H 12}^{*}=0.4899\left(s p^{2.01}\right)_{N}-0.8718(s)_{H}$} & \multirow{2}{*}{0.04429} & 002398 & 24 & 76 \\
\hline & $N_{11}-H_{12} \cdots O_{49}$ & $s p^{49.50}$ & 1.89247 & & & 0.02398 & 24 & 10 \\
\hline & $N_{44}-H_{45} \cdots O_{16}$ & $s p^{0.73}$ & 1.96322 & & & & & \\
\hline & $N_{44}-H_{45} \cdots O_{16}$ & $s p^{71.08}$ & 1.89247 & $\sigma_{N 44-H 45}=0.4897\left(s p^{2.01}\right)_{N}-0.8719(s)_{H}$ & 0.043397 & 0.023081 & 23.98 & 76.02 \\
\hline & $C_{17}-H_{19} \cdots C_{30}$ & $s p^{0.21}$ & 1.99144 & $\sigma_{C_{17}-H_{19}}^{*}=0.5988\left(s p^{2.15}\right)_{C}-0.8009(s)_{H}$ & 0.02306 & 0.00132 & 35.86 & 64.14 \\
\hline & & $s p^{99.99}$ & 1.96437 & & & & & \\
\hline & $C_{50}-H_{52} \cdots \mathrm{Cl}_{63}$ & $s p^{0.21}$ & 1.99133 & $\sigma_{C_{50}-H_{50}}^{*}=0.6062\left(s p^{2.33}\right)_{C}-0.7953(s)_{H}$ & 002208 & 000034 & 3675 & 6325 \\
\hline & $c_{50}-1152 \cdots l_{63}$ & $s p^{99.99}$ & 1.96472 & ${ }^{0} C_{50}-H_{52}-0.0002\left(\mathrm{SP} \quad \int_{C}-0.1300(3)_{H}\right.$ & 0.02200 & 0.00034 & 30.15 & 05.25 \\
\hline & $C_{51}-H_{53} \cdots N_{46}$ & $s p^{1.98}$ & 1.91916 & $\sigma_{C_{51}-H_{53}}^{*}=0.5967\left(s p^{2.45}\right)_{C}-0.8025(s)_{H}$ & 0.02547 & 0.00373 & 35.60 & 64.40 \\
\hline & $N_{11}-H_{12} \cdots O_{47}$ & $s p^{0.74}$ & 1.96142 & $\sigma_{N 11-H 12}^{*}=0.4893\left(s p^{2.02}\right)_{N}-0.8721(s)_{H}$ & 0.04391 & 0.02325 & 23.94 & 7606 \\
\hline & $N_{11}-H_{12} \cdots O_{47}$ & $s p^{62.96}$ & 1.89365 & & & & 2 & 10.00 \\
\hline $\mathrm{D}_{5}$ & $N_{42}-H_{43} \cdots O_{16}$ & $s p^{0.74}$ & 1.96142 & $\sigma_{N}^{*}$ & 0.04417 & 0.02351 & 23.98 & 7602 \\
\hline$D_{5}$ & $N_{42}-H_{43} \cdots O_{16}$ & $s p^{49.88}$ & 1.90488 & $0_{N 42-H 43}-0.403 /\left(\mathrm{SP} \quad \mathrm{N}_{N}-0.0 / 17(3)_{H}\right.$ & 0.07411 & 0.02531 & 25.70 & 10.02 \\
\hline & $C_{2}-H_{7} \cdots O_{47}$ & $s p^{0.74}$ & 1.96142 & $\sigma_{C 2-H 7}^{*}=0.6070\left(s p^{2.23}\right)_{C}-0.7947(s)_{H}$ & 0.01403 & 0.00035 & 37.02 & 62.98 \\
\hline & $C_{18}-H_{20} \cdots N_{13}$ & $s p^{1.97}$ & 1.92070 & $\sigma_{C 18-H 20}^{*}=0.5994\left(s p^{2.43}\right)_{C}-0.8004(s)_{H}$ & 0.02319 & 0.00115 & 35.93 & 64.07 \\
\hline
\end{tabular}

\subsubsection{Hyperconjugation and Rehybridization}

In $N-H \cdots O$ interactions, we have a rehybridization of the orbitals of nitrogen atoms with complexation. The orbitals of the nitrogen atoms are hybridized $s p^{2.20}$ in monomers while in dimers they are hybridized $s p^{n}(\mathrm{n} \in 2.01$ 2.03) (Table 7). The $P$ character of the orbitals of the nitrogen atoms decreases (the $\mathrm{S}$ character increases) with complexation. This observation is also verified by the parameter $\Delta \% S(X)$ of the nitrogen atoms of the $N-H \cdots O$ interaction which is between 1.73-2.46. This presence of rehybridization during complexation allows to shorten the $N-H$ bond [27]. The presence of hyperconjugative interactions $n_{O} \rightarrow \sigma_{N-H}^{*}$ with a stabilization energy between $5.17-11.31 \mathrm{kcal} / \mathrm{mol}$ for $N-H \cdots O$ interactions increases the electron density in the bonding orbitals $\sigma_{N-H}^{*}$ which are all polarized with respect to the hydrogen atom (Table 6), this polarization of the antibonding orbitals $\sigma_{N-H}^{*}$ with respect to the $\mathrm{H}$ atom ensures a better overlap $n_{O} \rightarrow \sigma_{N-H}^{*}$ [56]. The observed variation in electron density $\Delta \sigma^{*}$ has the effect of weakening the N-H bonds by lengthening them. The elongation of the N-H bond $(\Delta d(N-H)>0)$ is clearly demonstrated in the geometry of the complexes where it is between 10.19-11.8 $\mathrm{m} \AA$. Consequently, in the $N-H \cdots O$ interactions, the presence of the hyperconjugative interaction $n_{O} \rightarrow \sigma_{N-H}^{*}$ with high stabilization energy $E^{(2)}$ masks the hybridization effect, which leads to the elongation of $\mathrm{N}-\mathrm{H}$ bonds and a red shift in their vibrational stretching frequency [24, 27].

Except for the D2 dimer, $C-H \cdots O$ interactions are observed in the different complexes studied. carbon atoms (C) are hybridized $s p^{m}(\mathrm{~m} \in 2.26-2.28)$ in monomers and $s p^{2.23}$ in dimers. Also the variation in the percentage of the carbon atom $\Delta \% S(C)$ is between $0.29-0.51$. The $\mathrm{S}$ character of the carbon atoms involved in $C-H \cdots O$ interactions increases, so there is a presence of rehybridization with complexation. Hyperconjugative interactions $n_{O} \rightarrow \sigma_{N-H}^{*}$ are also observed in the D1 and D5 complexes with a low stabilization energy value of 0.06 and $0.05 \mathrm{kcal} / \mathrm{mol}$ respectively. the interactions $n_{O} \rightarrow \sigma_{N-H}^{*}$ were not observed in D3 and D5 dimers. For $C-H \cdots O$ interactions, the presence of hyperconjugative interaction with a very low value of $E^{(2)}$ and its absence in certain complexes (D3 and D5) do not allow to mask the effects of rehybridization observed with complexation. As a result, the $C-H$ bonds are shortened with a blue shift of their vibrational stretching frequency $[24,55]$. 
Table 7. Hybridization of the $X$ atoms involved in the $X-H \cdots Y$ interaction in the monomers and dimers and the variation of the $S$ character of the $X$ atom $(\Delta \% S(X))$ with the complexation.

\begin{tabular}{|c|c|c|c|c|}
\hline \multirow{2}{*}{ Complexes } & \multirow{2}{*}{ Contact } & \multicolumn{2}{|c|}{ Changes of hybridization $s p^{n}(X-H)$} & \multirow{2}{*}{$\Delta \% S(X)$} \\
\hline & & Monomer & Dimer & \\
\hline \multirow{8}{*}{ D1 } & $N_{11}-H_{12} \cdots O_{47}$ & $s p^{2.20}$ & $s p^{2.01}$ & 1.96 \\
\hline & $N_{42}-H_{43} \cdots O_{16}$ & $s p^{2.20}$ & $s p^{2.01}$ & 1.96 \\
\hline & $\mathrm{C}_{2}-\mathrm{H}_{7} \cdots \mathrm{O}_{47}$ & $s p^{2.28}$ & $s p^{2.23}$ & 0.51 \\
\hline & $C_{33}-H_{38} \cdots O_{16}$ & $s p^{2.28}$ & $s p^{2.23}$ & 0.51 \\
\hline & $C_{49}-H_{51} \cdots N_{44}$ & $s p^{2.45}$ & $s p^{2.23}$ & 0.15 \\
\hline & $C_{18}-H_{20} \cdots N_{13}$ & $s p^{2.45}$ & $s p^{2.23}$ & 0.15 \\
\hline & & $s p^{2.40}$ & $s p^{2.45}$ & -0.04 \\
\hline & $C_{17}-H_{19} \cdots_{27} H-C_{23}$ & $s p^{2.43}$ & $s p^{2.40}$ & -0.11 \\
\hline \multirow{2}{*}{ D2 } & $N_{11}-H_{12} \cdots O_{47}$ & $s p^{2.20}$ & $s p^{2.03}$ & 1.73 \\
\hline & $N_{42}-H_{43} \cdots O_{16}$ & $s p^{2.20}$ & $s p^{2.03}$ & 1.73 \\
\hline \multirow{6}{*}{ D3 } & $N_{44}-H_{45} \cdots O_{16}$ & $s p^{2.20}$ & $s p^{1.98}$ & 2.25 \\
\hline & $N_{11}-H_{12} \cdots O_{49}$ & $s p^{2.20}$ & $s p^{1.98}$ & 2.46 \\
\hline & $C_{18}-H_{20} \cdots N_{13}$ & $s p^{2.27}$ & $s p^{2.39}$ & 0.35 \\
\hline & $C_{2}-H_{7} \cdots O_{49}$ & $s p^{2.27}$ & $s p^{2.23}$ & 0.38 \\
\hline & & $s p^{2.29}$ & $s p^{2.28}$ & 0.1 \\
\hline & $C_{17}-H_{19} \cdots_{27} H-C_{23}$ & $s p^{2.41}$ & $s p^{2.43}$ & -0.11 \\
\hline \multirow{6}{*}{ D4 } & $N_{11}-H_{12} \cdots O_{49}$ & $s p^{2.20}$ & $s p^{2.01}$ & 1.94 \\
\hline & $N_{44}-H_{45} \cdots O_{16}$ & $s p^{2.20}$ & $s p^{2.01}$ & 1.9 \\
\hline & $C_{17}-H_{19} \cdots C l_{30}$ & $s p^{2.31}$ & $s p^{2.15}$ & 1.56 \\
\hline & $C_{50}-H_{52} \cdots C l_{63}$ & $s p^{2.31}$ & $s p^{2.33}$ & -0.11 \\
\hline & $C_{51}-H_{53} \cdots N_{46}$ & $s p^{2.31}$ & $s p^{2.45}$ & -1.2 \\
\hline & $C_{35}-H_{40} \cdots O_{16}$ & $s p^{2.26}$ & $s p^{2.23}$ & 0.29 \\
\hline \multirow{6}{*}{ D5 } & $N_{11}-H_{12} \cdots O_{47}$ & $s p^{2.20}$ & $s p^{2.02}$ & 1.9 \\
\hline & $N_{42}-H_{43} \cdots O_{16}$ & $s p^{2.20}$ & $s p^{2.01}$ & 1.96 \\
\hline & $C_{2}-H_{7} \cdots O_{47}$ & $s p^{2.28}$ & $s p^{2.23}$ & 0.42 \\
\hline & $C_{18}-H_{20} \cdots N_{13}$ & $s p^{2.45}$ & $s p^{2.43}$ & 0.16 \\
\hline & & $s p^{2.43}$ & $s p^{2.44}$ & 0.11 \\
\hline & $\mathrm{C}_{17}-H_{19} \cdots{ }_{27} \mathrm{H}-\mathrm{C}_{23}$ & $s p^{2.38}$ & $s p^{2.39}$ & -0.03 \\
\hline
\end{tabular}

$\Delta \% S(X)=\% S^{\text {dimer }}(X)-\% S^{\text {monomer }}(X)$

The $C-H \cdots N$ interactions are observed in D1, D3, D4 and D5 dimers. The hybridization states $s p^{m}$ of the carbon atoms involved in the $C-H \cdots N$ interactions reveal the presence of rehybridization with the complexation. We also note the presence of hyperconjugative interactions that allows an increase of the electron density in the antibonding orbital. This electron density $\Delta \sigma^{*}=0.000205$ e has the effect of lengthening the $\mathrm{CH}$ bond of $\Delta d(C-H)=0.00026 \AA$ in the interaction $\mathrm{C}_{18}-\mathrm{H}_{20} \cdots N_{13}$ combined with a red shift $\Delta v_{C-H}=-2.7588 \mathrm{~cm}^{-1}$ of the vibrational stretching frequency of the $C-H$ bond. However, in the D1, D4 and D5 dimers, the rehybridization which has the effect of lengthening the bonds during the complexation outweighs the hyperconjugative interactions. Therefore, we have an elongation of the $\mathrm{C}-\mathrm{H}$ bond with a blue shift of the vibrational stretching frequencies $[24,55]$. Concerning the $C-H \cdots H-C$ interactions, the hybridization states of the carbon atoms vary little with the complexation. In addition, NBO analysis did not detect the presence of interaction between orbitals in different bonds involved in $\mathrm{C}-\mathrm{H} \cdots \mathrm{H}-$ $C$ interactions. Consequently, a blue shift of the vibrational stretching frequencies is observed.

\subsubsection{AIM Analysis}

In the D1-D5 dimers, the $N-H \cdots O$ interactions have an electron density $\rho(r)$ between $0.024561-0.026517 e a_{0}^{-3}$ and a positive value of the Laplacian $\nabla^{2} \rho(r)$ (varying between $\left.0.082075-0.09015 e a_{0}^{-5}\right)$ and the total kinetic energy density $H(r)$ (varying between 0.000672-0.001158 ua). The values of $\rho(r), \nabla^{2} \rho(r)$ and $H(r)$ indicate a decrease of electronic density in the interatomic bond path characteristic of closedshell interactions such as hydrogen bonds [52, 54]. Also for all $N-H \cdots O$ interactions, the ratio $-\frac{G(r)}{V(r)}>1$ highlights the electrostatic nature of these hydrogen bonds [39-41]. Ellipticity is a measure of the stability of the chemical bond. A chemical bond with a large ellipticity is potentially unstable. The $N-H \cdots O$ interactions observed in dimers are stable because they have a low ellipticity $\varepsilon(r)$ in the range of 0.02064-0.031263. The Espinoza method was used to calculate the $N-H \cdots O$ interaction energies and the results obtained are shown in Table 8 . The energies of the $N-$ $H \cdots O$ interactions vary between $5.81074-6.29393 \mathrm{kcal} / \mathrm{mol}$. The $N-H \cdots O$ interactions are weak because the energies obtained are less than $15 \mathrm{kcal} / \mathrm{mol}$ [50]. 

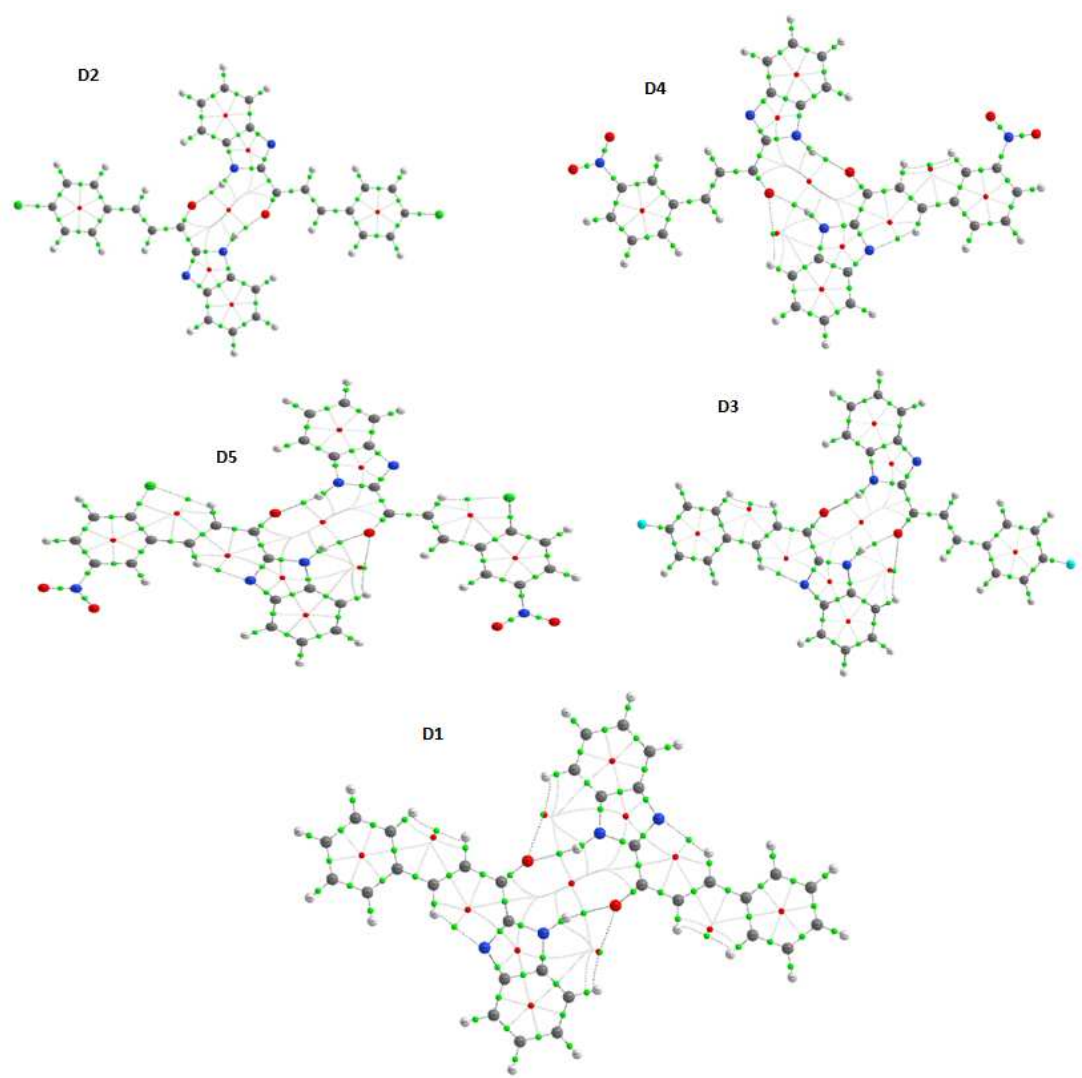

Figure 4. AIM molecular graphs of D1-D5 dimers at B3LYP 6-31+G $(d, p)$. Small green spheres are bond critical points (Bcps).

At the $C-H \cdots N$ interaction Bcps, the electronic density $\rho(r)$ is between $0.017788-0.01991 e a_{0}^{-3}$, the Laplacian of the electronic density $\nabla^{2} \rho(r)$ is positive and varies between 0.057507-0.062472 $e a_{0}^{-5}$. In addition, the total energy density $H(r)$ between $0.001253-0.001265$ au is positive. These features are typical of closed-shell interactions that indicate the presence of hydrogen bonding [52, 54]. In addition, all the interactions $C-H \cdots N$ have the ratio $-\frac{G(r)}{V(r)}$ greater than 1, thus highlighting the electrostatic nature of these bonds [41]. The $C-H \cdots N$ interactions are stable with an ellipticity $\varepsilon(r)$ between $0.003872-0.016963$. The interaction energies of $C-H \cdots N$ interactions range from $3.68662-4.22942 \mathrm{kcal} / \mathrm{mol}$ and are significantly lower than those of the $N-H \cdots O$ interactions.

The electron density obtained at the Bcps of the $C-$ $H \cdots O$ interactions is lower than those of the $N-H \cdots O$ and $C-H \cdots N$ interactions. This electron density $\rho(r)$ varies between $0.00228-0.00231904 e a_{0}^{-3}$, its Laplacian $\nabla^{2} \rho(r)$ is positive and varies between 0.009179-0.009449 $e a_{0}^{-5}$. Thus, a positive value of $H(r)$ is observed at the different Bcps of the $C-H \cdots O$ interactions. The values of $\rho(r), \nabla^{2} \rho(r)$ and $H(r)$ show that $C-H \cdots O$ interactions are weak hydrogen bonds $[52,54]$. Moreover, the ratio $-\frac{G(r)}{V(r)}>1$ for the $C-$ $H \cdots O$ interactions highlights its electrostatic nature [40, 41]. The high value of the ellipticity $\varepsilon(r)$ observed (1.51295711.458266) and the low value of the energy of the hydrogen bond $E_{H B} \quad(0.35141-0.36396 \mathrm{kcal} / \mathrm{mol})$ highlight their instabilities and weaknesses with respect to the $N-H \cdots O$ and $C-H \cdots O$ hydrogen bonds.

At the Bcps of the $C-H \cdots H-C$ interactions, the electronic density $\rho(r)$ is in the range of 0.008995 $0.009047 e a_{0}^{-3}$, the corresponding Laplacian is between $0.038854-0.039858 e a_{0}^{-5}$ with a positive energy density $H(r)(0.002478-0.002492 \mathrm{au})$. Also the interaction energy is about $1.49 \mathrm{kcal} / \mathrm{mol}$, thus $C-H \cdots H-C$ interactions are weak hydrogen bonds $[50,54]$. From more $-\frac{G(r)}{V(r)}>1$ at the Bcps of the different $C-H \cdots H-C$ interaction, thus the $C-H \cdots H-C$ interactions are purely electrostatic [40].

The ellipticity of the $C-H \cdots H-C$ interactions is between 1.145216-2.031056, so we can say that the $C-$ $H \cdots H-C$ interactions are less stable than the $C-H \cdots N$ and $N-H \cdots O$ interactions but more stable than the $C-$ $H \cdots O$ interaction.

Table 8. Topological analysis of the bond critical points (Bcps) of the $X-H \cdots Y$ interactions of D1-D5 dimers.

\begin{tabular}{|c|c|c|c|c|c|c|c|c|c|c|c|}
\hline & $X-H \cdots Y$ & & $\rho(\boldsymbol{r})_{X-H \cdots Y}$ & $\nabla^{2} \rho(r)_{X-H \cdots Y}$ & $\boldsymbol{V}(\boldsymbol{r})$ & $G(r)$ & $\boldsymbol{H}(\boldsymbol{r})$ & $L(\boldsymbol{r})$ & $\varepsilon(\boldsymbol{r})$ & $-\frac{G(r)}{V(r)}$ & $E_{H B}$ \\
\hline \multirow{4}{*}{ D1 } & $C_{17}-H_{19} \cdots_{27} H-C_{23}$ & intra & 0.009047 & 0.038856 & -0.00476 & 0.007235 & 0.002479 & -0.00971 & 1.1452 & 1.520 & 1.49347 \\
\hline & $C_{18}-H_{20} \cdots N_{13}$ & intra & 0.017788 & 0.057118 & -0.01175 & 0.013015 & 0.001265 & -0.01428 & 0.0170 & 1.108 & 3.68662 \\
\hline & $C_{49}-H_{51} \cdots N_{44}$ & intra & 0.017798 & 0.057149 & -0.01176 & 0.013023 & 0.001265 & -0.01429 & 0.0170 & 1.107 & 3.68976 \\
\hline & $C-H_{58} \cdots H-C$ & intra & 0.009047 & 0.038854 & -0.00476 & 0.007234 & 0.002478 & -0.00971 & 1.1457 & 1.520 & 1.49347 \\
\hline
\end{tabular}




\begin{tabular}{|c|c|c|c|c|c|c|c|c|c|c|c|}
\hline & $X-H \cdots Y$ & & $\rho(\boldsymbol{r})_{X-H \cdots Y}$ & $\nabla^{2} \rho(r)_{X-H \cdots Y}$ & $\boldsymbol{V}(\boldsymbol{r})$ & $G(r)$ & $H(r)$ & $L(r)$ & $\varepsilon(\boldsymbol{r})$ & $-\frac{G(r)}{V(r)}$ & $E_{H B}$ \\
\hline \multirow{6}{*}{ D2 } & $C_{2}-H_{7} \cdots O_{47}$ & inter & 0.00228 & 0.009426 & -0.00116 & 0.001755 & 0.0006 & -0.00236 & 11.4583 & 1.513 & 0.36396 \\
\hline & $N_{11}-H_{12} \cdots O_{47}$ & inter & 0.025987 & 0.087155 & -0.01972 & 0.020752 & 0.001036 & -0.02179 & 0.0300 & 1.052 & 6.18725 \\
\hline & $N_{42}-H_{43} \cdots O_{16}$ & inter & 0.025993 & 0.087181 & -0.01972 & 0.020758 & 0.001037 & -0.0218 & 0.0300 & 1.053 & 6.18725 \\
\hline & $C_{33}-H_{38} \cdots O_{16}$ & inter & 0.002281 & 0.009427 & -0.00116 & 0.001756 & 0.000601 & -0.00236 & 10.7918 & 1.514 & 0.36396 \\
\hline & $N_{11}-H_{12} \cdots O_{47}$ & inter & 0.026159 & 0.085744 & -0.01966 & 0.020549 & 0.000887 & -0.02144 & 0.0198 & 1.045 & 6.16842 \\
\hline & $N_{42}-H_{43} \cdots O_{16}$ & inter & 0.026159 & 0.085745 & -0.01966 & 0.020549 & 0.000887 & -0.02144 & 0.0198 & 1.045 & 6.16842 \\
\hline \multirow{5}{*}{ D3 } & $N_{44}-H_{45} \cdots O_{16}$ & inter & 0.025527 & 0.086416 & -0.01934 & 0.020472 & 0.001132 & -0.0216 & 0.0309 & 1.059 & 6.06802 \\
\hline & $N_{11}-H_{12} \cdots O_{49}$ & inter & 0.024561 & 0.079453 & -0.01852 & 0.019191 & 0.000672 & -0.01986 & 0.0206 & 1.036 & 5.81074 \\
\hline & $\mathrm{C}_{2}-\mathrm{H}_{7} \cdots \mathrm{O}_{49}$ & inter & 0.00224 & 0.009179 & -0.00112 & 0.001706 & 0.00059 & -0.0023 & 2.0833 & 1.523 & 0.35141 \\
\hline & $C_{35}-H_{40} \cdots O_{16}$ & Inter & 0.002252 & 0.009236 & -0.00113 & 0.001718 & 0.00059 & -0.00231 & 2.3831 & 1.520 & 0.35454 \\
\hline & $N_{44}-H_{45} \cdots O_{16}$ & Inter & 0.025138 & 0.082075 & -0.01898 & 0.019751 & 0.000767 & -0.02052 & 0.0212 & 1.041 & 5.95507 \\
\hline \multirow{6}{*}{ D4 } & $N_{11}-H_{12} \cdots O_{49}$ & Inter & 0.026517 & 0.090159 & -0.02006 & 0.021298 & 0.001242 & -0.02254 & 0.0313 & 1.062 & 6.29393 \\
\hline & $C_{17}-H_{19} \cdots C_{30}$ & intra & 0.014125 & 0.054501 & -0.00891 & 0.011268 & 0.002357 & -0.01363 & 0.0743 & 1.265 & 2.79556 \\
\hline & $C_{51}-H_{53} \cdots N_{46}$ & intra & 0.01991 & 0.062472 & -0.01348 & 0.014546 & 0.001071 & -0.01562 & 0.0039 & 1.079 & 4.22942 \\
\hline & $C_{50}-H_{52} \cdots C l_{63}$ & intra & 0.014913 & 0.055646 & -0.00961 & 0.011758 & 0.002153 & -0.01391 & 0.0388 & 1.224 & 3.01519 \\
\hline & $C_{18}-H_{20} \cdots N_{13}$ & intra & 0.017945 & 0.057507 & -0.01187 & 0.013124 & 0.001253 & -0.01438 & 0.0167 & 1.106 & 3.72427 \\
\hline & $C_{17}-H_{19} \cdots_{27} H-C_{23}$ & intra & 0.008995 & 0.038835 & -0.00473 & 0.007218 & 0.002492 & -0.00971 & 1.2077 & 1.526 & 1.48406 \\
\hline \multirow[t]{3}{*}{ D5 } & $N_{42}-H_{43} \cdots O_{16}$ & inter & 0.026125 & 0.08835 & -0.01977 & 0.02093 & 0.001158 & -0.02209 & 0.0304 & 1.059 & 6.20294 \\
\hline & $N_{11}-H_{12} \cdots O_{47}$ & inter & 0.024803 & 0.080458 & -0.01872 & 0.019418 & 0.000697 & -0.02012 & 0.0212 & 1.037 & 5.87349 \\
\hline & $C_{2}-H_{7} \cdots O_{47}$ & inter & 0.002319 & 0.009449 & -0.00115 & 0.001758 & 0.000604 & -0.00236 & 1.5130 & 1.529 & 0.36082 \\
\hline
\end{tabular}

\section{NCI-RDG Analysis}

In M1-M5 monomers and D1-D5 complexes, NCI analysis revealed the presence of hydrogen bonds, VDW interactions and repulsive interactions. The presence of the $N-H \cdots O$, $C-H \cdots N$ and $C-H \cdots O$ hydrogen bonds in the complexes is evidenced by the presence of peaks that appear at $-0,040<\operatorname{sign}\left(\lambda_{2}\right) \times \rho(r)<-0,015$ (figure 5 and 6 ). In figure 5 and 6 , the hydrogen bonds $N-H \cdots O, C-H \cdots N$ and $C-H \cdots O$ are characterized by the presence of blue isosurface between the atoms $\mathrm{H}$ and $\mathrm{O}, \mathrm{H}$ and $\mathrm{N}$. The presence of green isosurface reveals the presence of VDW interaction (figure 6). The peaks corresponding to these interactions are observed at $\operatorname{sign}\left(\lambda_{2}\right) \times \rho(r)=0$. Repulsive interactions are observed when we have the presence of a high electron density. In the $R D G=f\left(\operatorname{sign}\left(\lambda_{2}\right) \times \rho(r)\right)$ graphs, the peaks of repulsive interactions (steric hindrance) appear at $0,020 \prec \operatorname{sign}\left(\lambda_{2}\right) \times \rho(r) \prec 0,100$. The presence of these repulsive interactions is represented by red isosurfaces in the various benzene and imidazole ring.
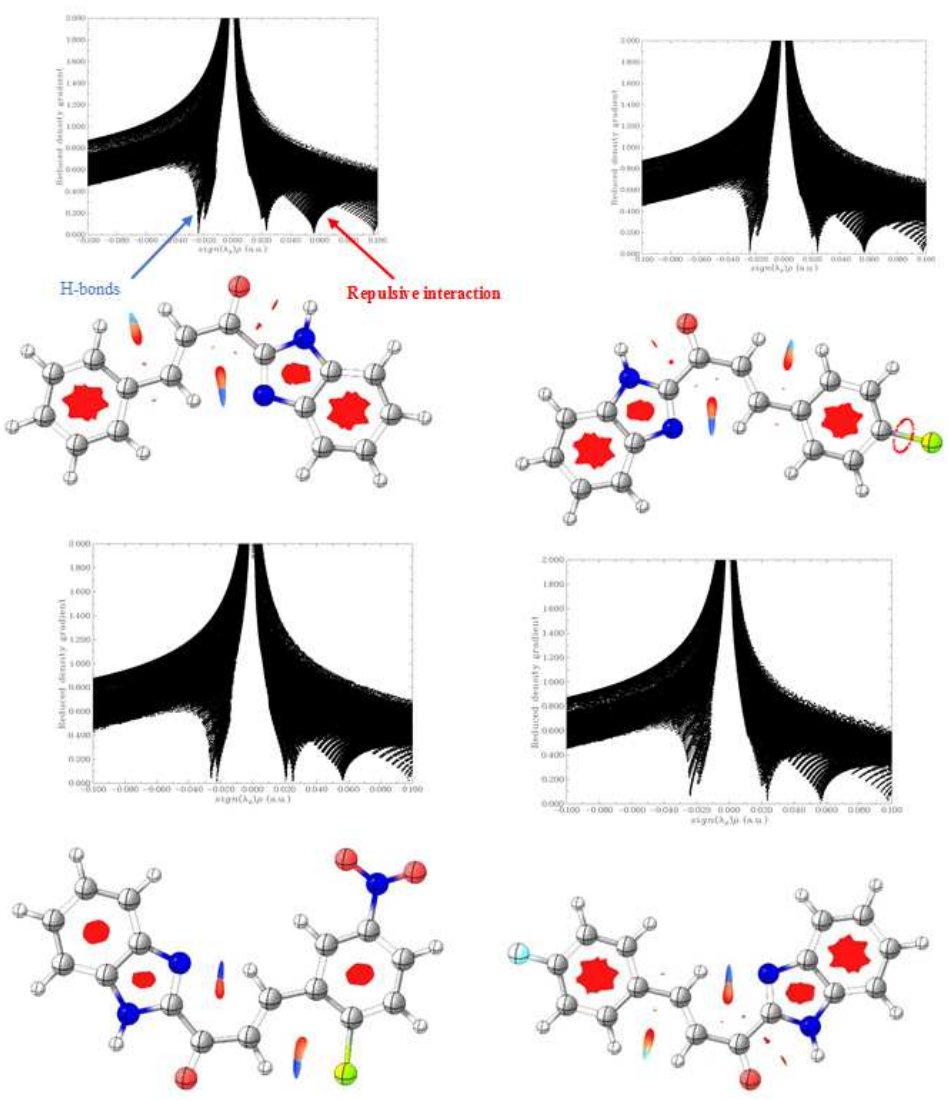

Figure 5. NCI isosurface and plot of the reduced density gradient $(R D G)$ versus sign $\left(\lambda \_2\right) \times \rho(r)$ of BZims monomer. 

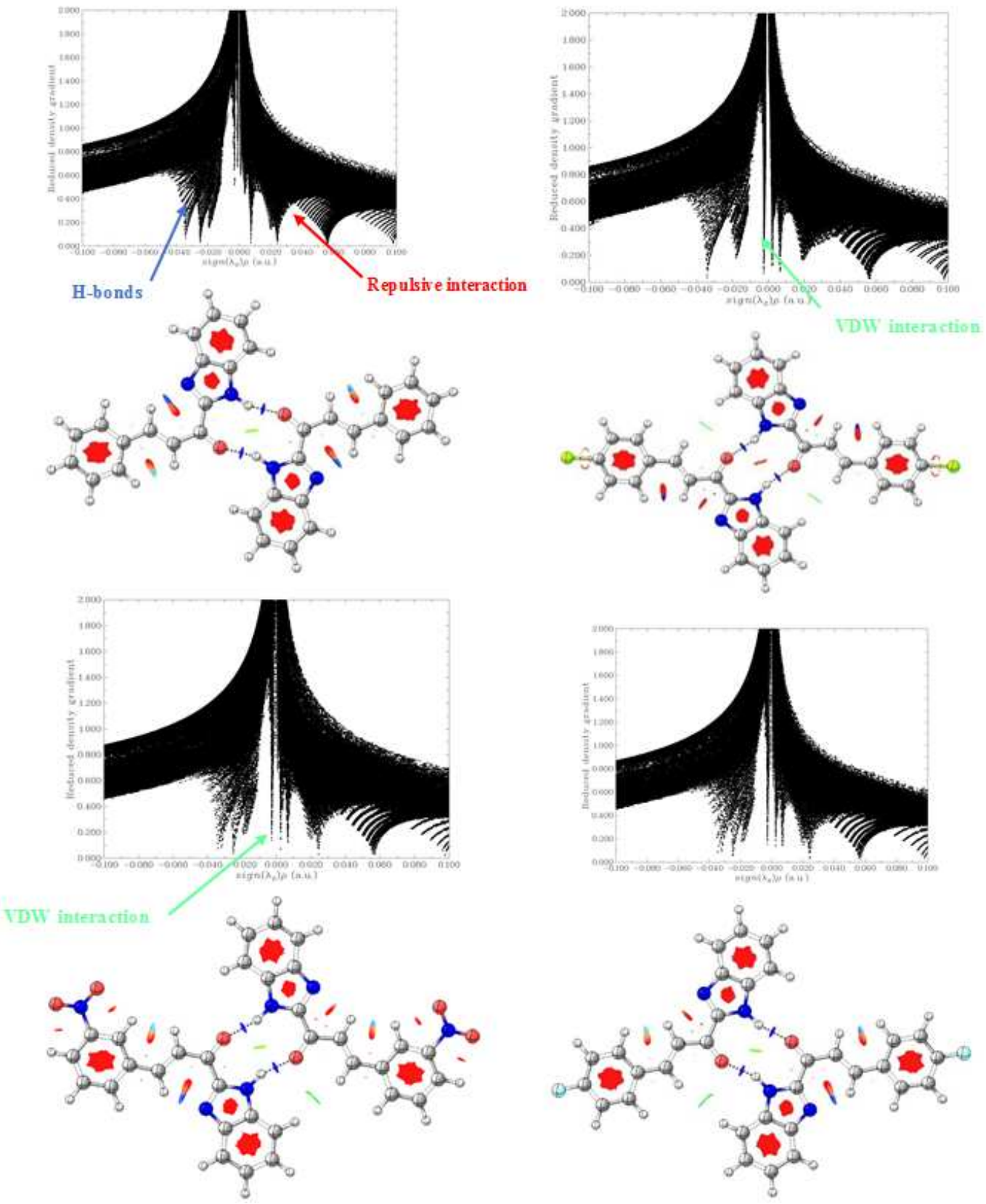

Figure 6. NCI isosurface and plot of the reduced density gradient $(R D G)$ versus $\operatorname{sign}\left(\lambda_{2}\right) \times \rho(r)$ of BZims complexes.

\section{Conclusion}

We carried out a detailed theoretical analysis of the monomers and dimers of BZims. Geometry optimization and harmonic frequency analysis were performed. The results of the frequency calculations show that all the monomers and dimers of the BZims are at least global structures. Our calculations clearly show that the BZims monomers and dimers have intramolecular and intermolecular hydrogen bonds. From the point of view of the interaction energy, the D2 dimer is more stable than the other forms. The stability sequence of dimers BZims is $D_{2}>D_{5}>D_{4}>D_{3}>D_{1}$. The presence of weakly electron acceptor group $(-\mathrm{Cl})$ on benzene ring favor the total interaction energy of dimerization. Evidence for the presence of intramolecular and intermolecular hydrogen bonding has been demonstrated by topological, geometrical, NCI and NBO analyzes. Frequency analysis results for BZims monomers and dimers indicate that the intramolecular hydrogen bond shows an increase in stretching frequencies and a blue shift in the IR spectrum, while the intermolecular hydrogen bond shows a decrease in stretching frequencies. and a red shift of the IR spectrum.

\section{References}

[1] Y. Wang, S. Xue, R. Li et al., "Synthesis and biological evaluation of novel synthetic chalcone derivatives as antitumor agents targeting Cat $\mathrm{L}$ and Cat K," Bioorganic \& medicinal chemistry, vol. 26, no. 1, pp. 8-16, 2018. 
[2] Z. M. Nofal, E. A. Soliman, S. S. Abd El-Karim et al., "Synthesis of Some New Benzimidazole-Thiazole Derivatives as Anticancer Agents," Journal of Heterocyclic Chemistry, vol. 51, no. 6, pp. 1797-1806, 2014.

[3] L.-t. Wu, Z. Jiang, J.-j. Shen et al., "Design, synthesis and biological evaluation of novel benzimidazole-2-substituted phenyl or pyridine propyl ketene derivatives as antitumour agents," European journal of medicinal chemistry, vol. 114, pp. 328-336, 2016.

[4] Ş. Demirayak, U. Abu Mohsen, and A. Çağri Karaburun, "Synthesis and anticancer and anti-HIV testing of some pyrazino[1,2-a]benzimidazole derivatives," European journal of medicinal chemistry, vol. 37, no. 3, pp. 255-260, 2002.

[5] Ş. Demirayak and L. Yurttaş, "Synthesis and anticancer activity of some 1,2,3-trisubstituted pyrazinobenzimidazole derivatives," Journal of enzyme inhibition and medicinal chemistry, vol. 29, no. 6, pp. 811-822, 2014.

[6] H. Göker, C. Kuş, D. W. Boykin et al., "Synthesis of some new 2-substituted-phenyl-1H-benzimidazole-5-carbonitriles and their potent activity against candida species," Bioorganic \& medicinal chemistry, vol. 10, no. 8, pp. 2589-2596, 2002.

[7] H. Göker, S. Ozden, S. Yildiz et al., "Synthesis and potent antibacterial activity against MRSA of some novel 1,2disubstituted-1H-benzimidazole-N-alkylated-5-

carboxamidines," European journal of medicinal chemistry, vol. 40, no. 10, pp. 1062-1069, 2005.

[8] T. Pan, X. He, B. Chen et al., "Development of benzimidazole derivatives to inhibit HIV-1 replication through protecting APOBEC3G protein," European journal of medicinal chemistry, vol. 95 , pp. 500-513, 2015.

[9] A. K. Tewari and A. Mishra, "Synthesis and Antiviral Activities of N-Substituted-2-substituted-benzimidazole Derivatives," ChemInform, vol. 37, no. 23, p. 489, 2006.

[10] H. A. BARKER, R. D. SMYTH, H. WEISSBACH et al., "Isolation and properties of crystalline cobamide coenzymes containing benzimidazole or 5, 6-dimethylbenzimidazole," The Journal of biological chemistry, vol. 235, pp. 480-488, 1960.

[11] Y. Yao, Y. Che, and J. Zheng, "The Coordination Chemistry of Benzimidazole-5,6-dicarboxylic Acid with Mn(II), Ni(II), and $\mathrm{Ln}(\mathrm{III})$ Complexes ( $\mathrm{Ln}=\mathrm{Tb}, \mathrm{Ho}, \mathrm{Er}, \mathrm{Lu}),{ }^{\prime}$ Crystal Growth \& Design, vol. 8, no. 7, pp. 2299-2306, 2008.

[12] D. Olea-Román, A. Solano-Peralta, G. Pistolis et al., "Lanthanide coordination compounds with benzimidazolebased ligands. luminescence and EPR," Journal of Molecular Structure, vol. 1163, pp. 252-261, 2018.

[13] N. Şırec1, Ü. Yilmaz, H. Küçükbay et al., "Synthesis of 1substituted benzimidazole metal complexes and structural characterization of dichlorobis(1-phenyl-1 H -benzimidazole$\kappa N 3)$ cobalt(II) and dichlorobis (1-phenyl-1 H -benzimidazolekN3)zinc(II)," Journal of Coordination Chemistry, vol. 64, no. 11, pp. 1894-1902, 2011.

[14] J. Kulhánek and F. Bureš, "Imidazole as a parent $\pi$-conjugated backbone in charge-transfer chromophores," Beilstein journal of organic chemistry, vol. 8, pp. 25-49, 2012.

[15] F. Saczewski, E. Dziemidowicz-Borys, P. J. Bednarski et al., "Synthesis, crystal structure and biological activities of copper(II) complexes with chelating bidentate 2-substituted benzimidazole ligands," Journal of inorganic biochemistry, vol. 100, no. 8, pp. 1389-1398, 2006.

[16] P. Gilli, V. Bertolasi, V. Ferretti et al., "Evidence for resonanceassisted hydrogen bonding. 4. Covalent nature of the strong homonuclear hydrogen bond. Study of the O-H--O system by crystal structure correlation methods," Journal of the American Chemical Society, vol. 116, no. 3, pp. 909-915, 1994.

[17] P. Gilli, V. Bertolasi, L. Pretto et al., "Covalent versus electrostatic nature of the strong hydrogen bond: Discrimination among single, double, and asymmetric singlewell hydrogen bonds by variable-temperature X-ray crystallographic methods in beta-diketone enol RAHB systems," Journal of the American Chemical Society, vol. 126, no. 12, pp. 3845-3855, 2004.

[18] S. Scheiner, Hydrogen bonding: A theoretical perspective, Oxford University Press, New York, 1997.

[19] P. Gilli, V. Bertolasi, V. Ferretti et al., "Evidence for Intramolecular $\mathrm{N}-\mathrm{H} \cdots \mathrm{O}$ Resonance-Assisted Hydrogen Bonding in $\beta$-Enaminones and Related Heterodienes. A Combined Crystal-Structural, IR and NMR Spectroscopic, and Quantum-Mechanical Investigation," Journal of the American Chemical Society, vol. 122, no. 42, pp. 1040510417, 2000.

[20] F. Cipcigan, V. Sokhan, G. Martyna et al., "Structure and hydrogen bonding at the limits of liquid water stability," Scientific reports, vol. 8, no. 1, p. 1718, 2018.

[21] J. Liu, X. He, J. Z. H. Zhang et al., "Hydrogen-bond structure dynamics in bulk water: Insights from ab initio simulations with coupled cluster theory," Chemical science, vol. 9, no. 8, pp. 2065-2073, 2018.

[22] C. Fonseca Guerra, F. M. Bickelhaupt, J. G. Snijders et al., "The Nature of the Hydrogen Bond in DNA Base Pairs: The Role of Charge Transfer and Resonance Assistance," Chemistry - A European Journal, vol. 5, no. 12, pp. 35813594, 1999.

[23] S. J. Grabowski, "What is the covalency of hydrogen bonding?," Chemical reviews, vol. 111, no. 4, pp. 2597-2625, 2011.

[24] I. V. Alabugin, Stereoelectronic effects: A bridge between structure and reactivity, Wiley, Chichester, West Sussex, UK, Hoboken, NJ, USA, 2016.

[25] A. S. Hansen, L. Du, and H. G. Kjaergaard, "The effect of fluorine substitution in alcohol-amine complexes," Physical chemistry chemical physics: PCCP, vol. 16, no. 41, pp. 22882-22891, 2014.

[26] P. Hobza and Z. Havlas, "Blue-Shifting Hydrogen Bonds," Chemical Reviews, vol. 100, no. 11, pp. 4253-4264, 2000.

[27] I. V. Alabugin, M. Manoharan, S. Peabody et al., "Electronic basis of improper hydrogen bonding: A subtle balance of hyperconjugation and rehybridization," Journal of the American Chemical Society, vol. 125, no. 19, pp. 5973-5987, 2003.

[28] R. G. Parr, "Density Functional Theory of Atoms and Molecules," in Horizons of Quantum Chemistry: Proceedings of the Third International Congress of Quantum Chemistry Held at Kyoto, Japan, October 29 - November 3, 1979, K. Fukui and B. Pullman, Eds., pp. 5-15, Springer Netherlands, Dordrecht, 1980. 
[29] J. K. Labanowski and J. Andzelm, Density functional methods in chemistry, Springer New York, New York, N. Y., 1991.

[30] Gaussian 09, Revision D.01 M. J. Frisch, G. W. Trucks, H. B. Schlegel, G. E. Scuseria, M. A. Robb, J. R. Cheeseman, G. Scalmani, V. Barone, B. Mennucci, G. A. Petersson, H. Nakatsuji, M. Caricato, X. Li, H. P. Hratchian, A. F. Izmaylov, J. Bloino, G. Zheng, J. L. Sonnenberg, M. Hada, M. Ehara, K. Toyota, R. Fukuda, J. Hasegawa, M. Ishida, T. Nakajima, Y. Honda, O. Kitao, H. Nakai, T. Vreven, J. A. Montgomery, Jr., J. E. Peralta, F. Ogliaro, M. Bearpark, J. J. Heyd, E. Brothers, K. N. Kudin, V. N. Staroverov, T. Keith, R. Kobayashi, J. Normand, K. Raghavachari, A. Rendell, J. C. Burant, S. S. Iyengar, J. Tomasi, M. Cossi, N. Rega, J. M. Millam, M. Klene, J. E. Knox, J. B. Cross, V. Bakken, C. Adamo, J. Jaramillo, R. Gomperts, R. E. Stratmann, O. Yazyev, A. J. Austin, R. Cammi, C. Pomelli, J. W. Ochterski, R. L. Martin, K. Morokuma, V. G. Zakrzewski, G. A. Voth, P. Salvador, J. J. Dannenberg, S. Dapprich, A. D. Daniels, O. Farkas, J. B. Foresman, J. V. Ortiz, J. Cioslowski, and D. J. Fox, Gaussian, Inc., Wallingford CT, 2013.

[31] AIMAll (Version 10.05.04), Todd A. Keith, 2010 (aim.tkgristmill.com).

[32] Glendening, A. E. Reed, J. E. Carpenter et al., "NBO Version 3.1," NBO Version 3.1.

[33] T. Lu and F. Chen, "Multiwfn: a multifunctional wavefunction analyzer," Journal of computational chemistry, vol. 33 , no. 5, pp. 580-592, 2012.

[34] chemcraft, Zhurko, G. A. and Zhurko, D. A. Chemcraft. Version 1.8 (Build 523a).

[35] S. F. Boys and F. Bernardi, "The calculation of small molecular interactions by the differences of separate total energies. Some procedures with reduced errors," Molecular Physics, vol. 19, no. 4, pp. 553-566, 1970.

[36] F. Weinhold and C. R. Landis, "Natural Bond Orbitals and extensions of localized bonding concepts," Chem. Educ. Res. Pract., vol. 2, no. 2, pp. 91-104, 2001.

[37] R. F. W. Bader, Atoms in molecules: A quantum theory, Clarendon Press; Oxford University Press, Oxford England, New York, 1994.

[38] I. Rozas, I. Alkorta, and J. Elguero, "Behavior of Ylides Containing $\mathrm{N}, \mathrm{O}$, and $\mathrm{C}$ Atoms as Hydrogen Bond Acceptors," Journal of the American Chemical Society, vol. 122, no. 45, pp. 11154-11161, 2000.

[39] Y.-Z. Yang, X.-F. Liu, R.-B. Zhang et al., "Joint experimental and theoretical studies of the surprising stability of the aryl pentazole upon noncovalent binding to $\beta$-cyclodextrin," Physical chemistry chemical physics: PCCP, vol. 19, no. 46, pp. 31236-31244, 2017.

[40] T. S. Koritsanszky, "Topology of X-Ray Charge Density of Hydrogen Bonds," in Hydrogen bonding-new insights, S. J. Grabowski, Ed., pp. 441-470, Springer, Dordrecht, 2006.

[41] R. Bianchi, G. Gervasio, and D. Marabello, "Experimental Electron Density Analysis of Mn 2 (CO) 10: Metal-Metal and Metal-Ligand Bond Characterization," Inorganic Chemistry, vol. 39, no. 11, pp. 2360-2366, 2000.
[42] E. Espinosa, E. Molins, and C. Lecomte, "Hydrogen bond strengths revealed by topological analyses of experimentally observed electron densities," Chemical Physics Letters, vol. 285, 3-4, pp. 170-173, 1998.

[43] B. Vijaya Pandiyan, P. Deepa, and P. Kolandaivel, "Do resonance-assisted intramolecular halogen bonds exist without a charge transfer and a $\sigma$-hole?," Physical chemistry chemical physics: PCCP, vol. 17, no. 41, pp. 27496-27508, 2015.

[44] E. R. Johnson, S. Keinan, P. Mori-Sánchez et al., "Revealing noncovalent interactions," Journal of the American Chemical Society, vol. 132, no. 18, pp. 6498-6506, 2010.

[45] J. Contreras-García, E. R. Johnson, S. Keinan et al., "NCIPLOT: a program for plotting non-covalent interaction regions," Journal of chemical theory and computation, vol. 7, no. 3, pp. 625-632, 2011.

[46] A. J. Cohen, P. Mori-Sánchez, and W. Yang, "Insights into current limitations of density functional theory," Science (New York, N. Y.), vol. 321, no. 5890, pp. 792-794, 2008.

[47] G. Saleh, C. Gatti, L. Lo Presti et al., "Revealing noncovalent interactions in molecular crystals through their experimental electron densities," Chemistry (Weinheim an der Bergstrasse, Germany), vol. 18, no. 48, pp. 15523-15536, 2012.

[48] D. N. Lande, S. A. Bhadane, and S. P. Gejji, "Noncovalent Interactions Accompanying Encapsulation of Resorcinol within Azacalix4pyridine Macrocycle," The journal of physical chemistry. A, vol. 121, no. 8, pp. 1814-1824, 2017.

[49] T. Steiner, "The Hydrogen Bond in the Solid State," Angewandte Chemie International Edition, vol. 41, no. 1, pp. 48-76, 2002.

[50] G. R. Desiraju and T. Steiner, The weak hydrogen bond: In structural chemistry and biology, Oxford University Press, Oxford etc., 1999.

[51] U. Koch and P. L. A. Popelier, "Characterization of C-H-O Hydrogen Bonds on the Basis of the Charge Density," The Journal of Physical Chemistry, vol. 99, no. 24, pp. 97479754, 1995.

[52] P. L. A. Popelier, Atoms in molecules: An introduction, Prentice Hall, Harlow, 2000.

[53] C. F. Matta, "Hydrogen-Hydrogen Bonding: The NonElectrostatic Limit of Closed-Shell Interaction Between Two Hydro," in Hydrogen bonding-new insights, S. J. Grabowski, Ed., pp. 337-375, Springer, Dordrecht, 2006.

[54] C. F. Matta and R. J. Boyd, The Quantum Theory of Atoms in Molecules, Wiley, 2007.

[55] P. Hobza and Z. Havlas, "Improper, blue-shifting hydrogen bond," Theoretical Chemistry Accounts: Theory, Computation, and Modeling (Theoretica Chimica Acta), vol. 108, no. 6, pp. 325-334, 2002.

[56] F. Weinhold and C. R. Landis, "Supramolecular bonding," in Valency and Bonding: A Natural Bond Orbital DonorAcceptor Perspective, F. WEINHOLD and C. R. LANDIS, Eds., pp. 579-709, Cambridge University Press, Cambridge, 2005. 